\title{
Long-Lived Ensembles of Shallow NV- Centers in Flat and Nanostructured Diamonds by Photoconversion
}

\author{
Federico Gorrini,* Carla Dorigoni, Domingo Olivares-Postigo, Rakshyakar Giri, Pietro Aprà, \\ Federico Picollo, and Angelo Bifone
}

Cite This: ACS Appl. Mater. Interfaces 2021, 13, 43221-43232

Read Online

ABSTRACT: Shallow, negatively charged nitrogen-vacancy centers $\left(\mathrm{NV}^{-}\right)$in diamond have been proposed for high-sensitivity magnetometry and spin-polarization transfer applications. However, surface effects tend to favor and stabilize the less useful neutral form, the $\mathrm{NV}^{0}$ centers. Here, we report the effects of green laser irradiation on ensembles of nanometer-shallow NV centers in flat and nanostructured diamond surfaces as a function of laser power in a range not previously explored (up to $150 \mathrm{~mW} / \mu \mathrm{m}^{2}$ ). Fluorescence spectroscopy, optically detected magnetic resonance (ODMR), and charge-photoconversion detection are applied to characterize the properties and dynamics of $\mathrm{NV}^{-}$and $\mathrm{NV}^{0}$ centers. We demonstrate that high laser power strongly promotes photoconversion of $\mathrm{NV}^{0}$ to $\mathrm{NV}^{-}$centers. Surprisingly, the excess $\mathrm{NV}^{-}$population is stable over a timescale of $100 \mathrm{~ms}$ after switching off the laser, resulting in long-lived enrichment of shallow $\mathrm{NV}^{-}$. The beneficial effect of photoconversion is less marked in nanostructured samples. Our results are important

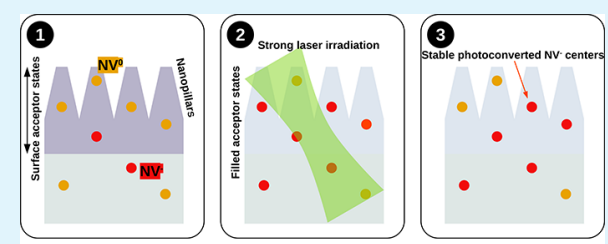
to inform the design of samples and experimental procedures for applications relying on ensembles of shallow $\mathrm{NV}^{-}$centers in diamond.

KEYWORDS: diamond, nitrogen-vacancy centers, $N V^{0}$, photoconversion, nanostructures, surface effects

\section{INTRODUCTION}

Negatively charged nitrogen-vacancy $\left(\mathrm{NV}^{-}\right)$centers are solidstate defects in the diamond lattice whose properties have been exploited to detect temperature gradients, ${ }^{1,2}$ magnetic $^{3,4}$ and electric fields ${ }^{5,6}$ at the nanoscale, and interactions with magnetic molecules and nanoparticles. ${ }^{7-10}$ Due to their biocompatibility, $\mathrm{NV}^{-}$-enriched fluorescent nanodiamonds represent promising sensors to investigate the cellular microenvironment in living tissues and their use in highsensitivity bioassays has been proposed. ${ }^{11-13}$ Furthermore, NV centers can be used in dynamic nuclear polarization (DNP) protocols where the polarization of the $\mathrm{NV}^{-} \mathrm{s}$ is transferred to ${ }^{13} \mathrm{C}$ nuclei, leading to hyperpolarization of ${ }^{13} \mathrm{C}$ nuclei in the diamond lattice. ${ }^{14-16}$ Substantial efforts are ongoing to promote polarization transfer from shallow $\mathrm{NV}$ centers to molecules absorbed at the diamond surface, ${ }^{17}$ thus enabling hyperpolarization of high-sensitivity tracers for biomedical magnetic resonance imaging.

For all these applications, the proximity of $\mathrm{NV}^{-}$centers to the diamond surface, where NVs can effectively interact with spins outside the diamond lattice, is of paramount importance, as the coupling strength between magnetic dipoles decreases with increasing distance. To this end, specially engineered layers of shallow $\mathrm{NV}^{-} \mathrm{s}^{18,19}$ as well as nanodiamonds ${ }^{20}$ have been proposed.
Unfortunately, surface states and defects at and close to the diamond surface can affect the charge stability of NV centers, reducing the availability of magnetically active $\mathrm{NV}^{-}$centers in favor of the neutral form ( $\mathrm{NV}^{0}$ centers), which do not present the same detection features. The relative stability and interconversion between the neutral and negatively charged states of the NV centers have been the object of investigation in several studies, ${ }^{21-25}$ and various attempts have been made to increase the stability of $\mathrm{NV}^{-}$centers, for instance, by surface termination, ${ }^{26}$ by doping of the diamond lattice, ${ }^{27}$ or by application of an electric field. ${ }^{28}$

Here, we investigate the effects of laser power and surface structures on charge stability and attainable spin polarization of shallow $\mathrm{NV}^{-}$centers in high-purity diamonds. Specifically, we aim to establish experimental conditions that maximize the availability of magnetically active $\mathrm{NV}^{-}$at the diamond surface. To this end, we apply fluorescence spectroscopy, as well as optically detected magnetic resonance (ODMR), in electronic-

Received: May 27, 2021

Accepted: August 2, 2021

Published: September 1, 2021 
grade diamond samples implanted with nitrogen ions at different depths and with flat or nanostructured surfaces.

Importantly, we study the effects of these factors in a regime of high-power laser irradiation not previously explored, and we assess the time dependence of the laser-induced $\mathrm{NV}^{-} / \mathrm{NV}^{0}$ distribution during and after irradiation.

\section{MATERIAL AND METHODS}

Sample Fabrication. We used four electronic-grade, single-crystal diamond plates, with an initial nitrogen concentration lower than 5 ppb (Element Six Technologies Ltd). The samples were $2.0 \times 2.0$ $\mathrm{mm}^{2}, 0.5 \mathrm{~mm}$ thick, with $\{100\}$ face orientation, and they were double-side polished, with roughness $R_{\mathrm{a}}<5 \mathrm{~nm}$ on both sides.

Two of these samples were nanostructured to increase the surfaceto-volume ratio. Samples nanostructuring was carried out using inductively coupled plasma-reactive ion etching (ICP-RIE) with oxygen gas, with a noncontinuous gold layer as a mask. ${ }^{29}$ Gold was deposited by DC sputtering (KS500 confocal sputtering system; Kenosistec Srl), using Ar gas (Figure 1a). Etching was performed by means of ICP-RIE (SI500-Sentech Instruments GmbH), using 700 $\mathrm{W}$ ICP power, $50 \mathrm{~W}$ plate power, and $40 \mathrm{sccm} \mathrm{O}_{2}$, at $1 \mathrm{~Pa}$ pressure, for $60 \mathrm{~s}$ (Figure 1b). After the ICP-RIE process, gold was removed with a commercial gold etchant (Sigma-Aldrich). Scanning electron microscopy (SEM) shows a tight arrangement of nanostructures ("nanopillars") (Figure 1e) over the diamond surface, with a height of $\approx 150 \mathrm{~nm}$. SEM images were acquired before gold removal.

Samples were implanted with ${ }^{15} \mathrm{~N}$ (INNOVION Corp., San Jose, CA) at a fluence of $10^{13} \mathrm{~cm}^{-2}$, at $7^{\circ}$ angle from normal to avoid ionchanneling ${ }^{30-32}$ (Figure 1c), in two different energy conditions: one flat sample and one nanostructured sample were implanted at $10 \mathrm{keV}$; the other flat and nanostructured ones were implanted at $20 \mathrm{keV}$ (see Table 1). Ion average ranges in the flat samples calculated by SRIM (http://www.srim.org, SRIM-2013.00) are, respectively, $\approx 15 \mathrm{~nm}$, with straggling of $5 \mathrm{~nm}$, and $\approx 27 \mathrm{~nm}$ with straggling of $8.5 \mathrm{~nm} .^{33}$

We then annealed the four samples at a temperature of $850{ }^{\circ} \mathrm{C}$ for $2 \mathrm{~h}$ in high vacuum conditions $\left(10^{-6} \mathrm{mbar}\right)$ (Figure $1 \mathrm{~d}$ ). The setup employed consists of a vacuum chamber equipped with a dry pumping system to avoid hydrocarbon contamination and a resistive heater. The heating element is a tantalum box, $5 \times 5 \times 5 \mathrm{~mm}^{3}$ in size, that keeps the sample in thermal equilibrium with the surrounding blackbody radiation. The temperature was externally monitored through an optical window using a pyrometer, pointed to the tantalum box. The heating and cooling rates were of $10{ }^{\circ} \mathrm{C} / \mathrm{min}$ to avoid thermal stresses in the diamond structures. At this temperature, vacancies and interstitial nitrogen atoms become mobile but substitutional nitrogen atoms are fixed in their position. ${ }^{34}$

We expect deeper NV centers for the $20 \mathrm{keV}$ than for $10 \mathrm{keV}$ implantation energies on average. For a rough estimate of the density of NV centers in the flat samples, we consider a conversion efficiency of $\approx 1 \%$ of the substitutional nitrogen after electron irradiation and annealing $^{35}$ This corresponds to an NV areal density of $10^{3} \mu \mathrm{m}^{-2}$. Dividing by the width of the implanted region $(10 \mathrm{~nm}$ indicatively, the order of magnitude of nitrogen straggling), we find an NV density of $0.57 \mathrm{ppm}$. In the nanostructured samples, a more inhomogeneous distribution profile is expected, as the implantation occurs on an irregular profile.

The fabrication process for the nanostructured samples is summarized in Figure 1a-d. For flat samples, implantation and annealing procedures were the same as in the nanostructured samples but were performed on a flat surface.

Experimental Setup. Full fluorescence spectra of NVs were taken with a confocal micro-Raman setup (LabRam Aramis, Jobin-Yvon Horiba), equipped with a $532 \mathrm{~nm}$ DPSS laser and an air-cooled multichannel CCD detector. Laser power was attenuated by neutral filters with 0,1 , or 2 optical densities (OD), resulting in laser intensities of $41 \mathrm{~mW} / \mu \mathrm{m}^{2}$ (OD0), $4.1 \mathrm{~mW} / \mu \mathrm{m}^{2}$ (OD1), and 0.41 $\mathrm{mW} / \mu \mathrm{m}^{2}$ (OD2). When referring to full fluorescence spectra taken with the micro-Raman, we use interchangeably laser intensities or optical densities. (a)

(b)
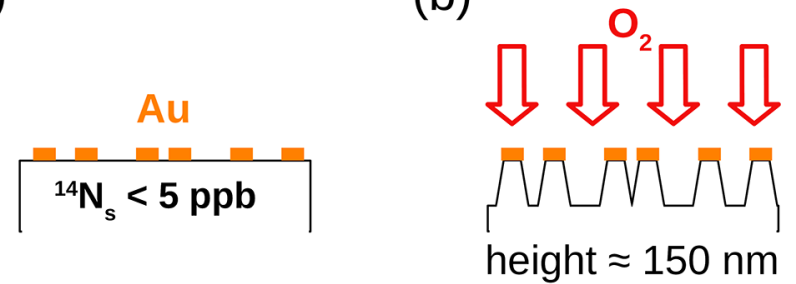

(c)

(d)

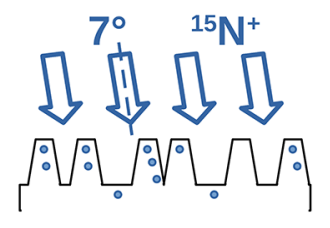

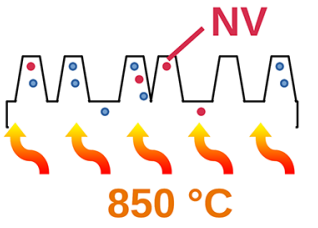

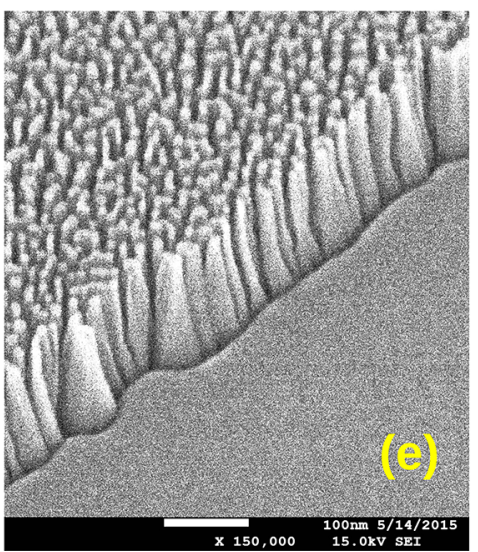

Figure 1. Nanofabrication process and synthesis of shallow NV centers in nanostructured samples. Electronic grade diamond with a very low concentration of nitrogen, less than $5 \mathrm{ppb}$, was masked with gold by sputtering (a) and then etched using oxygen reactive ion etching (b) to fabricate nanostructures, onto which nitrogen was implanted after gold removal (c). Samples were then annealed (d). SEM images show $\approx 150 \mathrm{~nm}$ high nanopillar-like structures (e). The SEM image was taken between steps (b) and (c), with still some conductive gold on the tips to improve image quality. Flat samples were implanted with ${ }^{15} \mathrm{~N}$ ions and then annealed, without application of any etching procedure.

Table 1. Type of Surfaces and Implantation Energies of ${ }^{15} \mathrm{~N}^{+}$ for the Four Electronic-Grade Samples Used in This Work

$\begin{array}{clc}\text { sample name } & \text { surface type } & \text { implantation energy }(\mathrm{keV}) \\ \text { F1 } & \text { flat } & 20 \\ \text { F2 } & \text { flat } & 10 \\ \text { N1 } & \text { nanostructured } & 20 \\ \text { N2 } & \text { nanostructured } & 10\end{array}$

Time-resolved measurements were performed with a home-built fluorescence microscope equipped with a green $532 \mathrm{~nm}$ laser $(5 \mathrm{~W}$, Verdi, Coherent) and a 0.25 NA objective (Plan N, Olympus). A focal spot size of $10 \mu \mathrm{m}^{2}$ was estimated. We used the same $532 \mathrm{~nm}$ laser to initialize and read out the state of the system. Fluorescence was collected by the objective and sent to a photon-counter module 
(a)

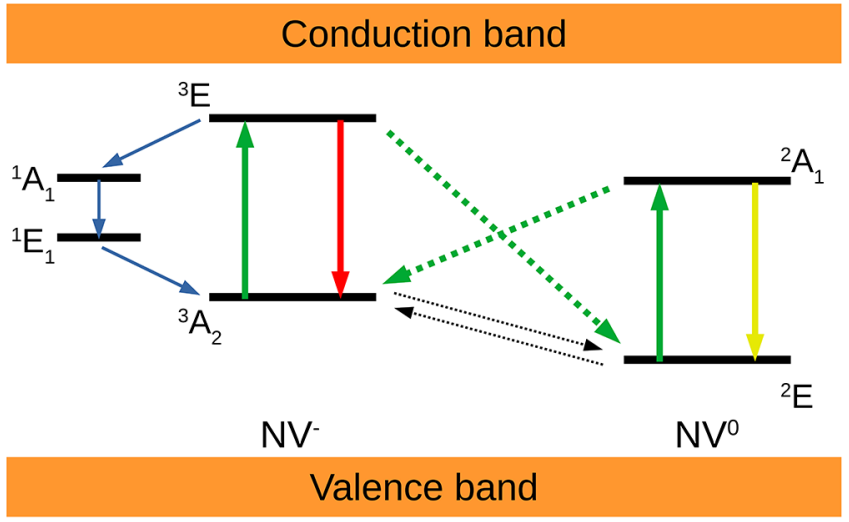

(c)

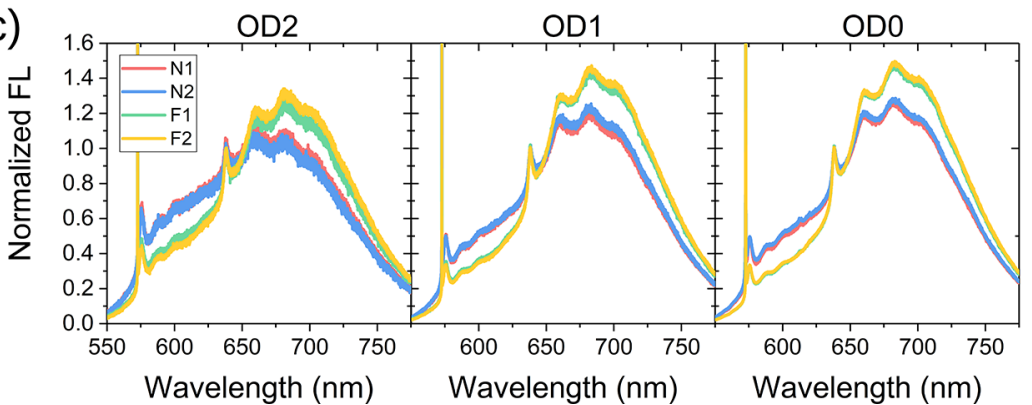

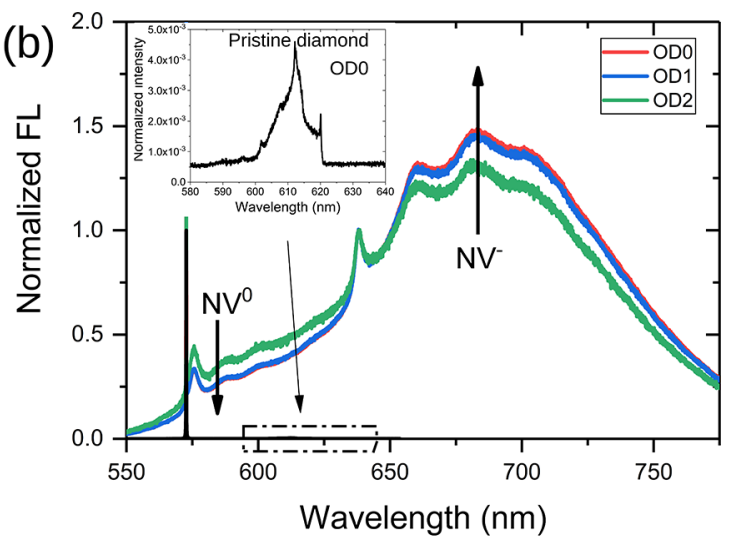

(d)

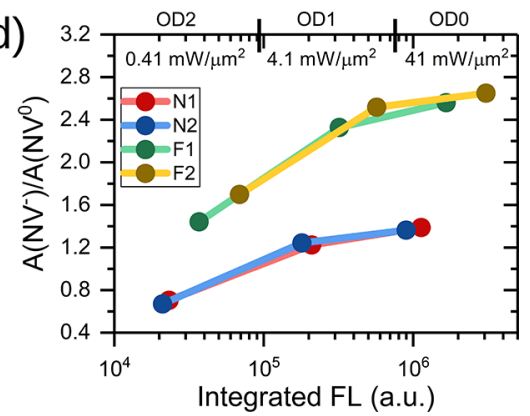

Figure 2. Fluorescence properties of $\mathrm{NV}$ centers. Electronic structures of $\mathrm{NV}^{-}$and $\mathrm{NV}^{0}$ centers are represented in (a). Green arrows indicate optical excitation and photoconversion (continuous and dotted arrows, respectively). Red and yellow arrows denote radiative decay from $\mathrm{NV}^{-}$and $\mathrm{NV}^{0}$ centers. Blue arrows indicate the nonradiative pathway, which leads to polarization of the $m_{\mathrm{s}}=0 \mathrm{NV}^{-}$ground state. Black arrows represent tunneling transitions in the dark between the two charged states. (b) Both $\mathrm{NV}^{-}$and $\mathrm{NV}^{0}$ fluorescence spectra were detected in implanted samples; no signal was detectable in nonimplanted, nonannealed samples (inset). Notably, emission from $\mathrm{NV}^{-} \mathrm{s}$ increases with laser power, at the expense of $\mathrm{NV}^{0}$ s. (c): the FL of nanostructured samples is lower compared to flat samples at all laser power levels (indicated by the filter optical density (OD)), with a larger component of $\mathrm{NV}^{0}$. The ratio of $\mathrm{NV}^{-}$and $\mathrm{NV}^{0} \mathrm{FL}$ intensities is plotted in (d), as a function of total integrated fluorescence and laser intensity. The $\mathrm{NV}^{-} / \mathrm{NV}^{0}$ ratio increases with laser power in all samples and is systematically larger in flat samples.

(Excelitas SPCM-AQRH-14-FC). Laser power at the sample surface was attenuated with a combination of absorptive filters and ranged from a maximum value of 1500 to $1 \mathrm{~mW}$, depending on the experiment's purpose. Correspondingly, the laser intensity could be tuned from a maximum of $150 \mathrm{~mW} / \mu \mathrm{m}^{2}$ to $100 \mu \mathrm{W} / \mu \mathrm{m}^{2}$. For nanostructured samples, irradiation occurred on an irregular "conical frustum" surface, with flat tops and regions between the structures, and tilted side surfaces. As a result, laser power (and laser intensity) should be downscaled by a factor of $2.5-3$. Even if this reduction in intensity may play a role, it cannot explain the large differences between flat and nanostructured samples reported in the Results section (differences are as if the laser power were 2 orders of magnitude less for the nanostructured samples). With the laser spot size calculated above, we estimated $10^{4} \mathrm{NVs}$ to be simultaneously excited by the laser.

We collected fluorescence through two different spectral windows, selected by combinations of filters: 550 to $600 \mathrm{~nm}$ (“550-600 nm") and $>750 \mathrm{~nm}$ ("750 nm+"). The $550-600 \mathrm{~nm}$ window is centered around the zero-phonon-line (ZPL) of the $\mathrm{NV}^{0}$ centers, at $575 \mathrm{~nm}$, and collects exclusively the $\mathrm{NV}^{0}$ signal. Conversely, the $>750 \mathrm{~nm}$ window is more selective for the $\mathrm{NV}^{-}$centers, even if a tail of the $\mathrm{NV}^{0}$ fluorescence can still leak through (see Section S1 of the Supporting Information).

We used a high-power acousto-optic modulator (ISOMET 523C6) for pulsed-laser sequences. Typically, a variable preparation pulse was applied to initialize the system, followed by a read-out pulse. A read-out pulse of $5 \mu$ s provided good sensitivity while minimally perturbing the distribution of NV charge states even at the highest laser power used (see Section S2 of the Supporting Information).

The microwave lines were composed of a microwave generator (Keysight N5171B), an amplifier (ZHL-16W-43-S+), and a millimeter-sized gold-coated copper loop placed below the sample.
A custom three-axis Helmholtz coil (Micro Magnetics, Inc) provided a static magnetic field along any desired direction. In some experiments, a strong magnetic field of $750 \mathrm{G}$ was applied along the $z$-axis, perpendicularly to the top diamond face, to quench the spin dynamics and enable selective measurement of charge dynamics. ${ }^{36}$ The $z$-axis was set parallel to the [100] crystallographic direction so that the applied magnetic field had the same magnitude along the four possible orientations of the $\mathrm{NV}$ centers axes in the diamond lattice.

\section{RESULTS}

Figure 2 shows the effects of laser power on the fluorescence (FL) spectra from different samples. The schematics of the energy level structures of $\mathrm{NV}^{0}$ and $\mathrm{NV}^{-}$centers are reported in Figure $2 \mathrm{a}$ to facilitate the interpretation of the experimental results. In short, NVs are characterized by a ground state (a spin-triplet ${ }^{3} \mathrm{~A}_{2}$ for $\mathrm{NV}^{-}$, and ${ }^{2} \mathrm{E}$ for $\mathrm{NV}^{0}$ ) and excited states $\left({ }^{3} \mathrm{E}\right.$ for $\mathrm{NV}^{-}$and ${ }^{2} \mathrm{~A}_{1}$ for $\left.\mathrm{NV}^{0}\right)$ positioned within the diamond band gap, ${ }^{37}$ each accompanied by a phonon band. Additionally, the $\mathrm{NV}^{-}$has two intermediate states $\left({ }^{1} \mathrm{~A}_{1}\right.$ and $\left.{ }^{1} \mathrm{E}\right)$ coupled to the ground and the excited states. The existence of this coupling enables a nonradiative transition that accumulates population in the $m_{\mathrm{s}}=0$ levels of the ground state under constant laser irradiation (blue arrows). A green laser (532 $\mathrm{nm}$ ) is used to excite both the $\mathrm{NV}^{-}$and the $\mathrm{NV}^{0}$ centers (green arrows). Laser irradiation can also induce a charge-state conversion, either by promoting an electron from the $\mathrm{NV}^{-}$ excited state into the conduction band $\left(\mathrm{NV}^{-} \rightarrow \mathrm{NV}^{0}\right.$ conversion) or by exciting an electron from the valence band 
(a)

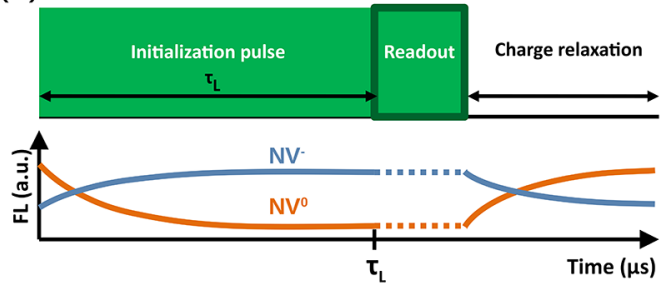

(b)

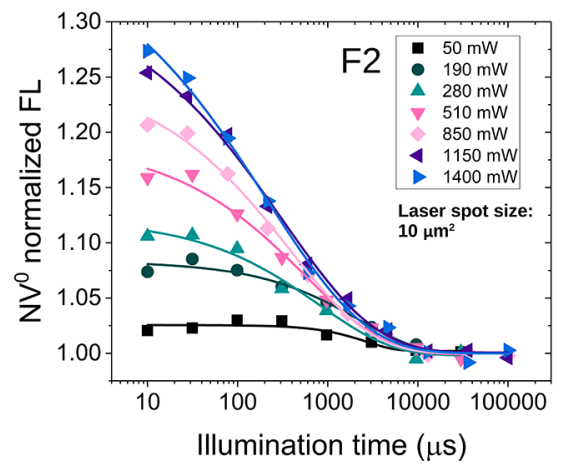

(c)

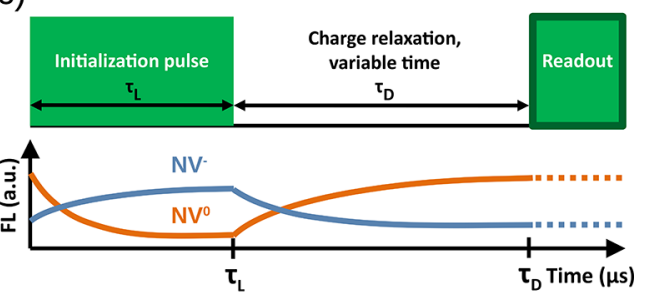

(d)

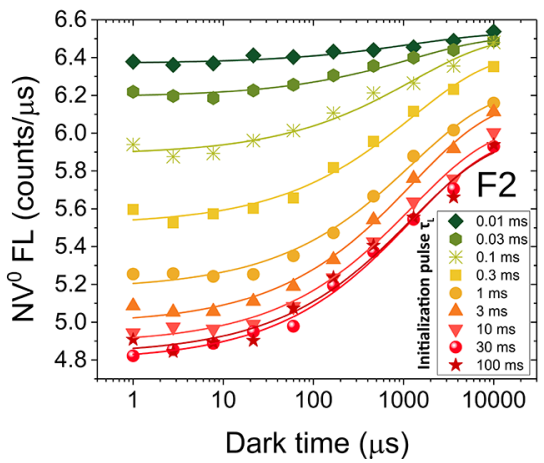

Figure 3. Detection of charge dynamics. (a) Pulse sequence adopted to investigate charge dynamics under laser pumping, for variable irradiation pulse length $\tau_{\mathrm{L}}$. Laser-induced $\mathrm{NV}^{0} \rightarrow \mathrm{NV}^{-}$photoconversion results in a decrease of the $\mathrm{NV}^{0}$ signal and an increase of the $\mathrm{NV}^{-}$. As an example, panel (b) shows the decrease in $\mathrm{NV}^{0} \mathrm{FL}$ for sample F2, as measured in the 550-600 nm window, for different laser powers. (c) Pulse sequence used to detect the charge recovery in the dark after a preparation pulse. Recovery of $\mathrm{NV}^{0} \mathrm{FL}$ for sample $\mathrm{F} 2$ at a laser power of $625 \mathrm{~mW}$ is shown in panel (d). Various curves in panel (d) correspond to different durations of the initialization pulse.

to the $\mathrm{NV}^{0}$ ground state $\left(\mathrm{NV}^{0} \rightarrow \mathrm{NV}^{-}\right.$conversion $){ }^{38}$ These two routes of photoconversion are represented by green dotted arrows. Experimental observations suggest that, upon switching off the laser, the system goes back to equilibrium through charge conversion in the dark, a process attributed to electron tunneling (black dotted arrows). Indeed, tunneling couples the $\mathrm{NV}$ centers to proximal electron donor or acceptor states, such as substitutional nitrogen atoms, vacancy complexes, and surface states. $23,25,39$

Figure $2 \mathrm{~b}$ shows fluorescence spectra from one sample (F2) at different laser powers. The NV charge states are characterized by different fluorescence spectra, with zerophonon lines (ZPL) at 575 and $638 \mathrm{~nm}$ for the $\mathrm{NV}^{0}$ and $\mathrm{NV}^{-}$, respectively, and a phonon sideband peaked around $620 \mathrm{~nm}$ for the $\mathrm{NV}^{0}$ and $700 \mathrm{~nm}$ for the $\mathrm{NV}^{-}$, and extending up to $\approx 800 \mathrm{~nm}$ in both cases. Spectral features from both $\mathrm{NV}^{-}$and $\mathrm{NV}^{0}$ centers are apparent, demonstrating the co-occurrence of the two charge states in this sample. The NV centers originate exclusively from implanted nitrogen (pristine diamond samples before implantation and annealing show no detectable fluorescence under a maximum laser intensity excitation of $41 \mathrm{~mW} / \mu \mathrm{m}^{2}$ but only the first- and second-order Raman peaks, shown in the inset). As the laser power intensity increases by 2 orders of magnitude (from $0.41 \mathrm{~mW} / \mu \mathrm{m}^{2}$ for filter optical density $\mathrm{OD}=2$ to $41 \mathrm{~mW} / \mu \mathrm{m}^{2}$ at $\left.\mathrm{OD}=0\right)$, the relative intensity of the $\mathrm{NV}^{-}$band increases with respect to that of the $\mathrm{NV}^{0}$ band. This indicates some degree of $\mathrm{NV}^{0} \rightarrow \mathrm{NV}^{-}$ photoconversion at higher laser powers under continuous excitation (curves are normalized to 1 at $638 \mathrm{~nm}$ ). The spectra in Figure $2 \mathrm{c}$ reflect the relative abundances of $\mathrm{NV}^{0}$ and $\mathrm{NV}^{-}$ centers in the nanostructured and flat samples for different laser powers. For the two nanostructured samples, the overall FL is lower (not apparent in the figure, where spectra are normalized for comparison), and the FL spectra show a higher component in the $\mathrm{NV}^{0}$ region (blue and red curves compared to yellow and green curves). In all samples, an increase in the $\mathrm{NV}^{-}$component and a decrease in $\mathrm{NV}^{0}$ is observed with increasing laser power. We summarize these observations in Figure $2 \mathrm{~d}$, where the $x$-axis represents the integrated total $\mathrm{FL}$, comprising the signals from both NV types, while the $y$-axis indicates the ratio of the areas under the $\mathrm{NV}^{-}$and $\mathrm{NV}^{0}$ curves. Fluorescence curves were deconvolved into $\mathrm{NV}^{0}$ and $\mathrm{NV}^{-}$ components before calculating the values of Figure $2 \mathrm{~d}$, similar to that in Alsid et al. ${ }^{40}$ (the process of integration and calculating the FL ratio is described in Section S1 of the Supporting Information). For all samples, the $\mathrm{NV}^{-} / \mathrm{NV}^{0}$ ratio increases with laser power, suggesting stronger $\mathrm{NV}^{0} \rightarrow \mathrm{NV}^{-}$ photoconversion at higher laser powers. Nanostructured samples (N1 and N2) contain fewer NV centers (reflected by an overall lower FL, integrated over the entire spectrum) and a higher fraction of $\mathrm{NV}^{0}$ compared to flat samples.

To explore the dynamics of laser-induced charge switching and subsequent return to equilibrium, we applied two pulsed experimental schemes (Figure 3). In these experiments, a strong magnetic field $(750 \mathrm{G})$ was applied along the [100] direction to suppress the effects of spin polarization of FL and selectively investigate charge dynamics. In fact, a sufficiently strong magnetic field $(>600 \mathrm{G})$ quenches the polarization of the $m_{\mathrm{s}}=0$ ground state by spin-state mixing and leads to a reduction of the spin-related component in the FL. ${ }^{36,41,42}$

Figure $3 \mathrm{a}, \mathrm{c}$ shows the two pulse sequences adopted to assess charge dynamics during laser irradiation and in the following dark time. In the first one (Figure 3a), a laser pulse $(532 \mathrm{~nm})$ of variable duration $\tau_{\mathrm{L}}$ (from $10 \mu \mathrm{s}$ to $100 \mathrm{~ms}$ ) was focused on the diamond surface to study charge photoconversion during irradiation. This pulse induces photoionization, with an increase in $\mathrm{NV}^{-}$and a decrease in the $\mathrm{NV}^{0}$ signal (blue and orange curves, respectively). FL was recorded by the subsequent short detection pulse of $5 \mu \mathrm{s}$. At the end of the sequence, a time interval of $10 \mathrm{~ms}$, during which the laser was 


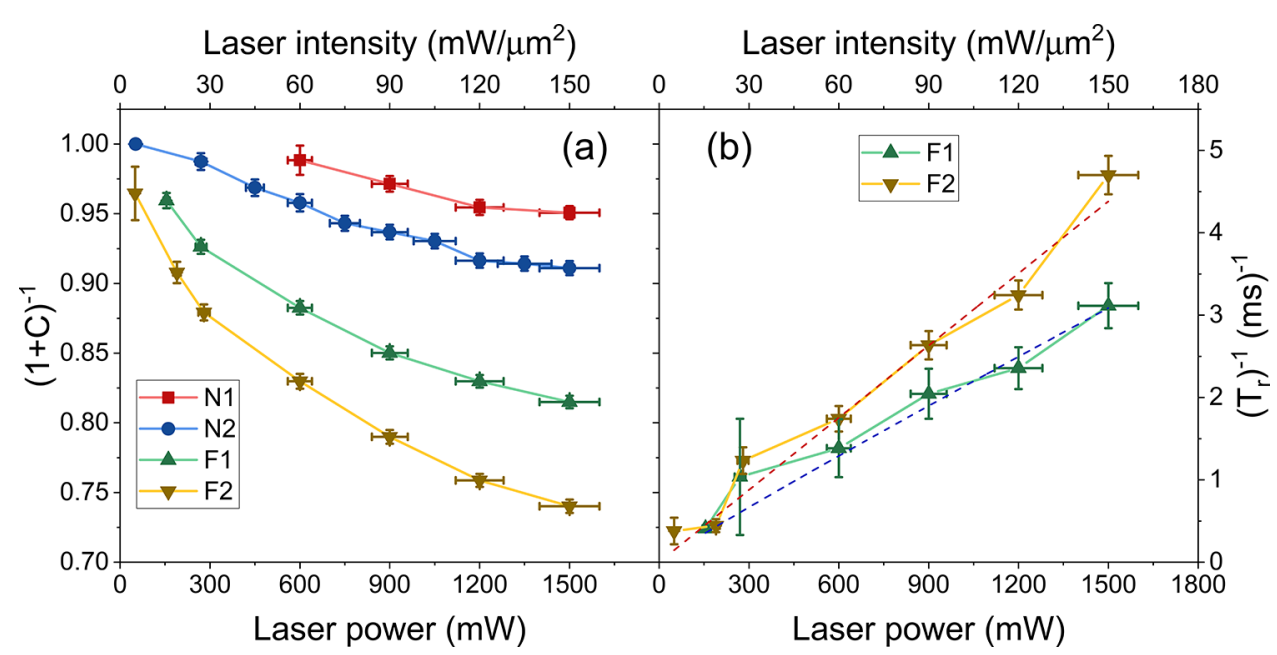

Figure 4. Parameters describing $\mathrm{NV}^{0} \rightarrow \mathrm{NV}^{-}$photoconversion under laser irradiation. The quantity $(1+C)^{-1}$ in $(\mathrm{a})$ indicates the ratio between the equilibrium value of FL and the initial value, right after the initialization pulse. The decrement is more consistent for the flat samples (down to $75 \%$ for sample F2). The photoconversion rate $\left(T_{\mathrm{r}}\right)^{-1}$ displayed in $(\mathrm{b})$ is nearly linear with laser power, following a power law with exponents of $0.95 \pm 0.07$ for F1 and $1 \pm 0.07$ for F2 (red and blue dashed curves).

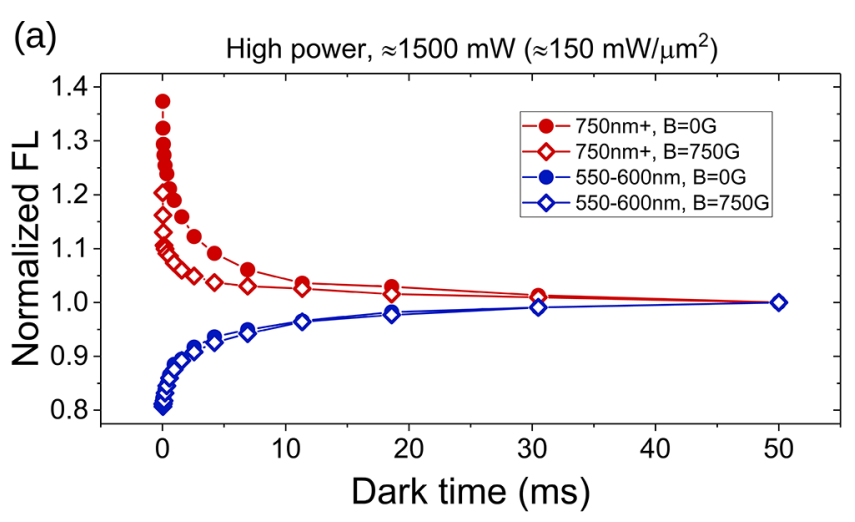

(b)

(c)

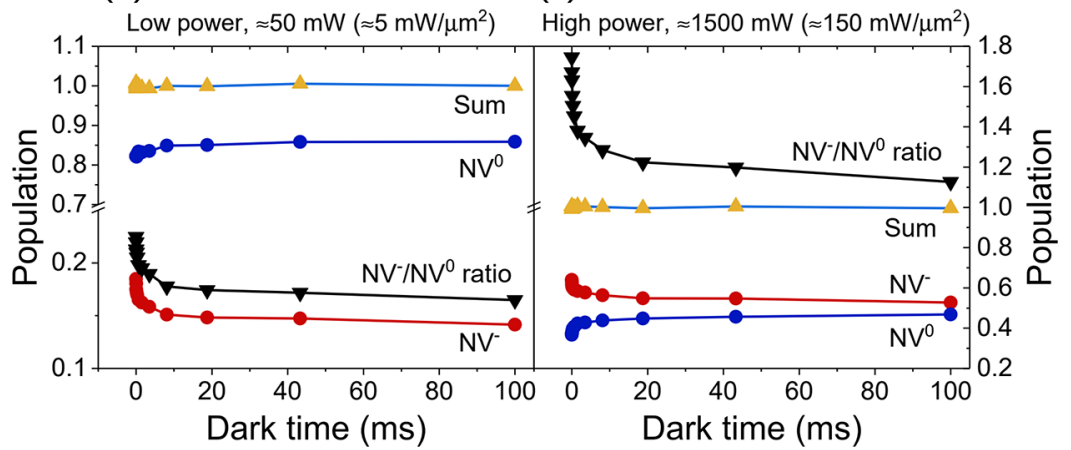

Figure 5. Spin and charge dynamics after the laser pulse. The effect of a strong magnetic field $(750 \mathrm{G})$ on the fluorescence of sample F2 at a laser power of $1500 \mathrm{~mW}$ is shown in (a). FL was collected in the $550-600 \mathrm{~nm}$ region (blue curves) and in the $>750 \mathrm{~nm}$ window (red curves), with and without magnetic field (empty and solid symbols, respectively). When considering only the $\mathrm{NV}^{0}$ centers $(550-600 \mathrm{~nm}$ window), no change in the FL profile is observed, irrespective of the magnetic field applied. On the contrary, the signal in the $>750 \mathrm{~nm}$ window is reduced by a strong magnetic field. This difference is the result of quenching $\mathrm{NV}^{-}$spin polarization through spin state mixing. However, beyond $\approx 10 \mathrm{~ms}$, the difference vanishes, and the evolution at longer times is dominated by charge dynamics. At low (b) and high (c) laser powers, the $10 \mathrm{~ms}$ laser pulse creates an excess of $\mathrm{NV}^{-} \mathrm{s}$ (red curve) that convert to $\mathrm{NV}^{0} \mathrm{~s}$ (blue curve) in the dark. $\mathrm{NV}^{-}$and $\mathrm{NV}^{0}$ populations are normalized such that their sum is 1 (yellow-azure curve). The ratio $R$ is indicated by the black curve. A near-equilibrium ratio (0.15) is reached after $100 \mathrm{~ms}$ at low power (50 mW), while a large and sustained $R(1.13)$ value is observed after high power irradiation $(1500 \mathrm{~mW})$. Results of (b) and (c) are taken at a magnetic field of $750 \mathrm{G}$, which enables determining the charge state ratio (for more details, see the text or Section S4 of the Supporting Information).

off, allowed for charge relaxation before the experiment was repeated for signal averaging. Data points acquired after discrete initialization pulses, for different values of laser power, are shown in Figure 3b (for sample F2). For simplicity, only
FL signals acquired in the range 550-600 nm, reflecting predominantly $\mathrm{NV}^{0}$ fluorescence, are reported (the behavior of $\mathrm{NV}^{-}$is symmetrical). The curves reported in this figure show that the degree of photoconversion increases steeply with 
lasers power, ranging between $50 \mathrm{~mW}$ to $1400 \mathrm{~mW}$. Independently of laser power, for pulses longer than $10 \mathrm{~ms}$, the fluorescence level lies within $5 \%$ of its equilibrium value, and photoconversion approaches saturation in all samples. Therefore, in the following experiments, we use an initialization pulse of at least $10 \mathrm{~ms}$ to maximize the number of $\mathrm{NV}^{-}$.

The second pulse sequence of Figure $3 c$ was used to probe the charge dynamics in the dark. The sequence consists of a laser pulse of duration $\tau_{\mathrm{L}}$ followed by a variable dark time $\tau_{\mathrm{D}}$ (from $1 \mu \mathrm{s}$ to $10 \mathrm{~ms}$ ) and a $5 \mu \mathrm{s}$ readout pulse. In Figure $3 \mathrm{~d}$, the FL of the $\mathrm{NV}^{0}$ centers measured through the 550-600 nm spectral window is reported as a function of $\tau_{\mathrm{D}}$. As discussed above, the first initialization pulse reduces the number of $\mathrm{NV}^{0}$ by photoconversion to $\mathrm{NV}^{-}$. During the dark time, the system goes back to equilibrium and a steady increase in the number of $\mathrm{NV}^{0}$ centers is reflected by increased fluorescence in the spectral region 550-600 $\mathrm{nm}$ registered by the readout pulse. A plausible explanation of this recharging-in-the-dark process, previously reported and discussed, ${ }^{23,25}$ contemplates electrons tunneling from the negative $\mathrm{NV}^{-}$to surface acceptors or vacancies until equilibrium is reached. To exemplify this phenomenon, Figure $3 \mathrm{~d}$ shows the curves obtained with a laser power of $\approx 625 \mathrm{~mW}$ and initialization times from $10 \mu \mathrm{s}$ to 100 ms for sample F2. Coherent with the results of Figure 3b, the initial value of fluorescence decreases with longer initialization pulses, meaning that an increasing fraction of $\mathrm{NV}^{0}$ is converted into $\mathrm{NV}^{-}$upon laser irradiation. In all cases, fluorescence from $\mathrm{NV}^{0}$ increases in the dark with longer $\tau_{\mathrm{D}}$. We also notice that the curves acquired after a $\tau_{\mathrm{L}}$ of 10,30 , and $100 \mathrm{~ms}$ are almost identical, consistent with the observation that beyond $10 \mathrm{~ms}$ the photoconversion process saturates.

The curves of Figure $3 b$ can be fitted with a stretched exponential law of the type ${ }^{8}$

$$
I(t)=I_{e q}\left[1+C \mathrm{e}^{-\left(t / T_{\mathrm{r}}\right)^{n}}\right]
$$

where $T_{\mathrm{r}}$ represents a characteristic timescale for the photoconversion process under the laser pulse, $n$ is the stretching factor, $I_{\mathrm{eq}}$ is the FL value at equilibrium, and $C$ is a positive parameter describing the fluorescence drop from the initial value to equilibrium. Curves were normalized by $I_{\mathrm{eq}}$ for easier comparison. Charge recovery curves, as in Figure 3d, can also be fitted with the function of eq 1 , even though, in this case, the pre-exponential parameter is negative (the initial value is lower than the equilibrium value). It should be noted that the mechanisms underlying charge conversion in the dark are different from those that induce photoconversion, so the two characteristic times $T_{\mathrm{r}}$ are not related.

The photoconversion data for all samples are reported in Figure $4 \mathrm{a}$, where we plot $(1+C)^{-1}$ (eq 1$)$, corresponding to the ratio between the FL equilibrium and initial values, for different laser powers. The largest drop in $\mathrm{NV}^{0} \mathrm{~s}$ due to photoconversion was observed in the flat samples (down to $74 \%$ for $\mathrm{F} 2$ and $81 \%$ for F1) at the largest available laser power, while more limited variation was observed in the nanostructured samples. Note that the large error bars on the $x$-axis are caused by a slight deterioration in the laser beam's transmittance at high laser power, an effect that accounts for $5-10 \%$ of losses during prolonged irradiation. In Figure $4 \mathrm{~b}$, we show the rate of photoconversion (i.e., the inverse of the photoconversion time $T_{\mathrm{r}}$ ) for the flat samples (in the case of nanostructured samples, the uncertainties on extracted values are too large to draw conclusions on their laser-power dependence). $\left(T_{\mathrm{r}}\right)^{-1}$ is seen to increase with laser power according to a power law with exponents of $0.95 \pm 0.07$ and 1 \pm 0.07 for samples F1 and F2, respectively (dashed lines). The nearly linear dependence on the laser power is suggestive of a single-photon-mediated $\mathrm{NV}^{0} \rightarrow \mathrm{NV}^{-}$photoconversion mechanism.

To illustrate the symmetry between the dynamics of the two charge states in the dark, in Figure 5a, we show the FL signal in the 550-600 and $>750 \mathrm{~nm}$ spectral windows, dominated by $\mathrm{NV}^{0}$ and $\mathrm{NV}^{-}$, respectively, for sample $\mathrm{F} 2$ at the highest laser power. The experiment of Figure $3 \mathrm{c}$ was performed with or without an applied magnetic field of $750 \mathrm{G}$ (open and solid symbols in Figure 5a, respectively). Indeed, while the evolution of the $\mathrm{NV}^{0}$ signal reflects strictly charge dynamics, the $\mathrm{NV}^{-}$ signal is influenced by both charge and spin dynamics. The applied magnetic field suppresses spin dynamics and makes it possible to selectively measure the effects of charge switching for $\mathrm{NV}^{-}$. Under these experimental conditions, the two curves, in red and blue, representing charge state recovery for $\mathrm{NV}^{-}$ and $\mathrm{NV}^{0}$, appear symmetrical, thus corroborating the idea that laser light converts one state into the other.

Performing the experiment of Figure $3 \mathrm{c}$ with the two 550600 and $>750 \mathrm{~nm}$ spectral windows makes it possible to estimate the charge-state ratio $R=\left[\mathrm{NV}^{-}\right] /\left[\mathrm{NV}^{0}\right]$ at a steady state, as proposed by Giri et al. ${ }^{36}$ (derivation of $R$ is illustrated in Section S4 of the Supporting Information). The two assumptions are (i) all the NV centers are optically active and (ii) the sum of $\mathrm{NV}^{-}$and $\mathrm{NV}^{0}$ is always constant. The latter hypothesis implies that a reduction of $\mathrm{NV}^{0}$ is counterbalanced by an increase of the $\mathrm{NV}^{-}$, and vice versa. Figure $5 \mathrm{~b}, \mathrm{c}$ shows the subdivision of the populations between $\mathrm{NV}^{-}$and $\mathrm{NV}^{0}$ (at $B=750 \mathrm{G}$ ) after a $10 \mathrm{~ms}$ laser pulse at low and high powers for sample F2, as an example. In both cases, the initialization pulse produces an excess of $\mathrm{NV}^{-}$centers that convert back into $\mathrm{NV}^{0}$ during the dark time. At low laser power $(50 \mathrm{~mW}, \approx 5 \mathrm{~mW} /$ $\mu \mathrm{m}^{2}$ ), the signal is dominated by $\mathrm{NV}^{0}$ s, with a ratio $R=0.13$ / $0.87 \approx 0.15$ at the longest time point acquired $(100 \mathrm{~ms})$. However, at high power $(1500 \mathrm{~mW}$, corresponding to $\approx 150$ $\mathrm{mW} / \mu \mathrm{m}^{2}$ ) the ratio changes to $0.53 / 0.47 \approx 1.13$, with the $\mathrm{NV}^{-}$centers now being the dominant state after a sevenfold increase compared to the low power case. Interestingly, after an initial rapid decrease, a large value of $R(1.13)$ is observed after $100 \mathrm{~ms}$, and the curve exhibits a much more slowly decaying component. Thus, the effect of an intense laser pulse is to create a large $\mathrm{NV}^{-} / \mathrm{NV}^{0}$ ratio that is sustained over tens or hundreds of milliseconds (see the Discussion section). Evidently, the charge dynamics has fast and slow components, summarized by the stretched exponential behavior, with the fast components in the $10-1000 \mu$ s timescale and the slower ones on a $100 \mathrm{~ms}$ scale (Figure $5 \mathrm{~b}, \mathrm{c}$ ). On the contrary, the timescale of spin dynamics is of the order of $\approx 1 \mathrm{~ms}$. For this reason, when considering the combined effect of spin and charge dynamics, there will be a substantial overlap in the fluorescence up to $1-10 \mathrm{~ms}$, while at longer times the FL profile will be dominated by charge dynamics. This is also apparent from Figure 5a, where the difference between the two red curves, attributed to spin dynamics, vanishes after $\approx 10 \mathrm{~ms}$; the evolution at longer times is unaffected by the magnetic field, thus indicating that it can be attributed exclusively to charge dynamics.

Finally, repeating the same experiments with sample N2 gives similar results, but with a modest degree of $\mathrm{NV}^{0} \rightarrow \mathrm{NV}^{-}$ 
(a)

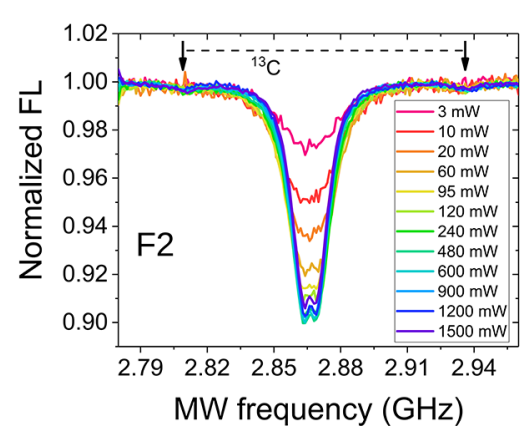

(b) Laser intensity $\left(\mathrm{mW} / \mu \mathrm{m}^{2}\right)$

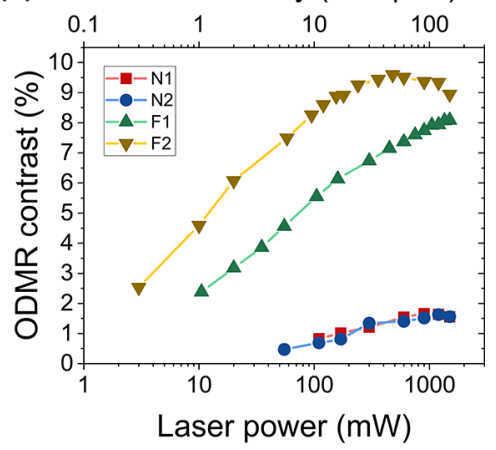

Figure 6. Optically detected magnetic resonance. (a) ODMR spectra from sample F2 as a function of laser power. (b) ODMR contrast for all samples. The increase, in contrast, is more pronounced for flat samples.

photoconversion and a shorter lifetime of the $\mathrm{NV}^{-}$-photoconverted centers (approximately $10 \mathrm{~ms}$ ). Even at the highest power of $1500 \mathrm{~mW}$, the ratio $\mathrm{NV}^{-} / \mathrm{NV}^{0}$ is $0.1 / 0.9 \approx 0.11$, much lower compared to the results of sample F2 (as shown in Figure S4 in Section S4).

Finally, we report optically detected magnetic resonance (ODMR) experiments to assess the spin polarization of the ground-state level $m_{\mathrm{s}}=0$ of $\mathrm{NV}^{-}$. Continuous laser irradiation polarizes the $m_{\mathrm{s}}=0$ state, while a sweeping microwave field transfers the population to the $m_{\mathrm{s}}= \pm 1$ state. However, we note that the detected ODMR contrast is also affected by $\mathrm{NV}^{0}$ centers. Indeed, even though FL is collected in the $>750 \mathrm{~nm}$ window, where most of the signal comes from the $\mathrm{NV}^{-}$ centers, a residual background signal from $\mathrm{NV}^{0} \mathrm{~s}$ is still present. This residual $\mathrm{NV}^{0}$ signal is not modulated by microwave irradiation and cannot be removed entirely by spectral filtering. Hence, we expect the ODMR contrast to be increased at higher laser power due to two independent mechanisms: (i) increased $\mathrm{NV}^{-}$polarization and (ii) reduction in the nuisance background signal from $\mathrm{NV}^{0}$.

In Figure 6a, we show the ODMR spectra from sample F2 for a fixed microwave power and increasing the laser power up to $1500 \mathrm{~mW}$. The contrast (i.e., the depth of the dip) increases rapidly with laser power and makes it possible to resolve the strain-split doublet ${ }^{43}$ and the ${ }^{13} \mathrm{C}$ sidebands at the highest contrast-to-noise ratio. In Figure $6 \mathrm{~b}$, we show the dependence of the ODMR contrast on laser power for all samples. Nanostructured samples exhibit low contrast and limited gain with increasing laser power, consistent with the evidence that $\mathrm{NV}^{0}$ remains the dominant charge state even at the highest laser powers. The ODMR contrast in flat samples is much larger and increases sharply with laser power. Saturation of contrast for the F2 sample at around $600 \mathrm{~mW}$ probably reflects laser-induced repolarization related to insufficient microwave power (see the Discussion section). In light of the above results, we can conclude that the increment in the ODMR contrast with laser power does not merely reflect increased ground-state spin polarization, but also a gain in the number density of $\mathrm{NV}^{-}$as a result of photoconversion (see the Discussion section for the consideration on the ODMR contrast).

\section{DISCUSSION}

Our results highlight the importance of $\mathrm{NV}^{0}$ to $\mathrm{NV}^{-}$charge conversion in shallow defects, and point to the use of highpower laser irradiation to improve the availability and polarization of shallow $\mathrm{NV}^{-}$. This effect appeared to be more prominent in flat samples, compared to nanostructured samples.

The maximum values of laser intensities used here are larger than those commonly reported in the literature. In fact, NV centers are typically investigated using laser intensities of few $\mathrm{mW} / \mu \mathrm{m}^{2}$ or less ${ }^{21,44,45}$ and only rarely approach $100 \mathrm{~mW} /$ $\mu \mathrm{m}^{2}{ }^{40}$ The use of such a high laser power is justified by the lack of saturation of the NV optical transition, as shown in Figure S3 (Section S3 of the Supporting Information). Indeed, we recorded FL emission in the two windows "550-600 nm" and ">750 nm" with increasing laser power: the signals from the $>750 \mathrm{~nm}$ window barely show any indication of saturation (saturation power estimated at $8.5 \mathrm{~W}$ ), while the $550-600 \mathrm{~nm}$ window shows a flexion only for the flat samples (saturation power estimated at $2.3 \mathrm{~W}$ ). Thus, we can conclude that the maximum laser power used here was below the onset of saturation. These results are not surprising, as saturation curves depend on whether a single NV center or ensembles are observed and on the sample characteristics (defects type and density). Very different saturation intensities are reported in the literature, ranging from a few $\mathrm{mW} / \mu \mathrm{m}^{246-48}$ to an estimate of thousands of $\mathrm{mW} / \mu \mathrm{m}^{2}$. ${ }^{49}$

In all four samples investigated, the $\mathrm{NV}^{0}$ centers represent a substantial proportion of NV defects. In the bulk, the presence of electron donors, such as substitutional nitrogen, favors the formation and stability of the $\mathrm{NV}^{-}$center. However, moieties and acceptor states at the diamond surface decrease the stability of the $\mathrm{NV}^{-}$, even at depths of tens of nanometers, ${ }^{50-52}$ and $\mathrm{NV}^{0} \mathrm{~s}$ may become the dominant charge state ${ }^{53,54}$ with detrimental effects for sensing and polarization transfer applications. Our samples were engineered to present shallow NV centers (similar to the previous reports ${ }^{55}$ ), and it is not surprising to find predominantly $\mathrm{NV}^{0}$ centers. In the case of nanostructured samples, the surface effects are exacerbated by the large surface-to-volume ratio of the nanostructures. Therefore, diamonds with nanopillars have fewer $\mathrm{NV}^{-} \mathrm{s}$ compared to $\mathrm{NV}^{0} \mathrm{~s}$, as apparent in the FL and ODMR spectra (Figures 2c, 4a, and $6 \mathrm{~b}$, respectively).

Moreover, the integrated fluorescence of Figure $2 \mathrm{~d}$ suggests that nanostructured samples have a lower number of NV centers altogether, independent of the specific charge state. This could be determined by less efficient ion implantation on the nanostructures or by a loss of nitrogen due to ion straggling.

Laser irradiation has two effects on the NV centers. The first effect is the polarization of the $\mathrm{NV}^{-} m_{\mathrm{s}}=0$ spin state. The 
second effect is to convert part of the $\mathrm{NV}^{0} \mathrm{~s}$ into $\mathrm{NV}^{-}$and increase the ratio of the negatively charged centers over the total NVs. Upon switching the laser off, a process of recharging in the dark results in an $\mathrm{NV}^{-} \rightarrow \mathrm{NV}^{0}$ back-conversion. An interesting finding of this work is that the excess $\mathrm{NV}^{-}$ population is persistent over a relatively long timescale.

The following hypothesis can qualitatively explain the experimental findings. A laser pulse ionizes the substitutional nitrogen atoms, ${ }^{56}$ and electrons are released into the conduction band and recombine with electron-trapping defects, ${ }^{57}$ including the $\mathrm{NV}^{0}$. The decrease of $\mathrm{NV}^{0} \mathrm{FL}$ in Figure $3 \mathrm{~b}$ is consistent with this mechanism. The phenomenon of redistribution of charges was recently investigated from a different perspective, namely the Stark shift of optical transition. ${ }^{39}$ The nearly-linear dependence of $\left(T_{\mathrm{r}}\right)^{-1}$ on laser power (Figure $4 \mathrm{~b}$ ) suggests a single-photon ionization process of substitutional nitrogen as a source of electrons for $\mathrm{NV}^{0} \rightarrow$ $\mathrm{NV}^{-}$photoconversion. We note that the proposed mechanism for $\mathrm{NV}^{0} \rightarrow \mathrm{NV}^{-}$charge-state conversion is different from the two-photon excitation of electrons from the valence band (i.e., a photon initializing the $\mathrm{NV}^{0}$ to the excited state and a second photon promoting an electron from the valence band to a trapping $\mathrm{NV}^{0}$ centers), ${ }^{38}$ which is better described by a quadratic dependence of $\left(T_{\mathrm{r}}\right)^{-1}$ on laser power. ${ }^{21}$ In the dark, an $\mathrm{NV}^{-} \rightarrow \mathrm{NV}^{0}$ back-conversion is observed. The mechanism proposed for this process is electron tunneling, which critically depends on the NV environment. Tunneling to a neighboring trap can only occur if the trap is empty. ${ }^{25}$ At weak laser powers, the photoexcited electrons do not saturate the traps in the vicinity of the $\mathrm{NV}$ center. At stronger laser powers, increasingly distant traps are populated. Hence, the time the system takes to return to equilibrium depends on laser power, as tunneling to distant traps is a much slower process. This picture is consistent with the results of Figure $5 b, c$ that show, at high laser power, a large $\left[\mathrm{NV}^{-}\right] /\left[\mathrm{NV}^{0}\right]$ ratio persistent on a scale of $100 \mathrm{~ms}$. Thus, high-power irradiation increases the availability of $\mathrm{NV}^{-}$and extends the time window in which the $\mathrm{NV}^{-}$spin states can be manipulated and detected, with important implications for sensing and polarization-transfer applications.

The beneficial effects of high-power irradiation are less pronounced in the etched samples, with a modest increase in $\mathrm{NV}^{-}$population. This is consistent with the idea that surface defects compete with $\mathrm{NV}^{0}$ to capture released electrons. Therefore, the $\mathrm{NV}^{0} \rightarrow \mathrm{NV}^{-}$conversion is more efficient in the flat samples and less efficient in the nanostructured ones, where the amount of surface defects is large compared to the amount of NVs (Figure 4a). In our samples, the advantage of the increased exposed area resulting from nanostructuring appears to be mitigated by the less favorable charge dynamics. Additional treatment of the surface to stabilize $\mathrm{NV}^{-}$(like selective oxidation ${ }^{58}$ or fluorination ${ }^{26}$ ) may improve the performance of nanostructured samples, but we have not explored the effects of different terminations in this paper. It is interesting to compare our results concerning nanopillars with recent work, ${ }^{59}$ where nanostructures demonstrated higher brightness, larger $\mathrm{NV}^{-} / \mathrm{NV}^{0}$ ratio, and better spin properties compared to a bulk reference. We can explain the differences by accounting for the striking different sizes of nanostructures. In the work of McCloskey, ${ }^{59}$ the nanopillars size was $\approx 1 \mu \mathrm{m}$ (as an order of magnitude for both height and width), while our nanostructures were much smaller, with a height of 150 $\mathrm{nm}$ and a width narrowing from $50 \mathrm{~nm}$ at the base to $10 \mathrm{~nm}$ at the top. Therefore, the effects of lateral surfaces are expected to be relevant for small nanostructures but negligible for micrometric pillars, where only a small fraction of the NV centers (the closest to the sidewall) is affected. Additional work would be necessary to trace the transition from nano- to microstructures in terms of $\mathrm{NV}^{-} / \mathrm{NV}^{0}$ ratio and to understand if the size and shape can affect the way photoexcited electrons recombine with defects, including $\mathrm{NV}^{0}$.

Interestingly, both routes of photoconversion $\left(\mathrm{NV}^{-} \rightarrow \mathrm{NV}^{0}\right.$ and $\mathrm{NV}^{0} \rightarrow \mathrm{NV}^{-}$) have been reported in the literature (see, for instance, refs 40, 44, 57, 60 for $\mathrm{NV}^{-} \rightarrow \mathrm{NV}^{0}$ and refs 25, 55 for $\mathrm{NV}^{0} \rightarrow \mathrm{NV}^{-}$). These different trends of charge-state conversion are related to the environment of the NV centers, i.e., to the presence of electron donors (mostly substitutional nitrogen) and electron acceptors (like vacancies created by nitrogen implantation or surface acceptors). Thus, the photostability and the routes of photoconversion are in general different for bulk and shallow NV centers, as suggested by the recent literature. ${ }^{36,54}$ Our results apply to shallow NV centers, which are relevant for sensing and polarization-transfer applications. Importantly, we stress the fact that in our samples a high laser power has a twofold beneficial effect: first, it increases the density of $\mathrm{NV}^{-} \mathrm{s}$ by photoconverting part of the $\mathrm{NV}^{0} \mathrm{~s}$, and second, it polarizes the $\mathrm{NV}^{-}$spins. This might not be the case for bulk samples, as improved spin polarization at high laser power could be frustrated by a loss of $\mathrm{NV}^{-}$centers via $\mathrm{NV}^{-} \rightarrow \mathrm{NV}^{0}$ photoionization.

An intermediate scenario between shallow $\mathrm{NV}$ centers and native bulk NV centers is represented by high-energy nitrogen implantation (hundreds of $\mathrm{keV}$ or $\mathrm{MeV}$ ). On the one hand, it has been reported that, under these conditions, the $\mathrm{NV}$ yield from nitrogen is extremely high (approximately 50\% for 2 $\mathrm{MeV}^{61}$ and $18 \mathrm{MeV}^{35}$ of implantation energy), so a high density of $\mathrm{NV}$ centers can be generated at a depth of $1 \mu \mathrm{m}$ or more, where the effects of surface acceptors on the $\mathrm{NV}^{-}$ stability are negligible. On the other hand, high implantation energies and doses are accompanied by the creation of a large number of vacancies, divacancies, and vacancy clusters, which might deteriorate the diamond lattice and compromise the $\mathrm{NV}^{-}$stability. ${ }^{62,63}$ The effect of nitrogen implantation energy and dose on $\mathrm{NV}^{-}$and $\mathrm{NV}^{0}$ charge dynamics might be the subject of a future analysis.

We used stretched exponentials to fit all the fluorescence curves to account for heterogeneity in the ensemble of NVs, resulting in a superposition of many single exponential curves. ${ }^{8,64}$ The dynamics of charge tunneling was addressed by Miller ${ }^{65}$ and Tachiya ${ }^{66}$ by considering a spatially homogeneous distribution of charge acceptors and recently extended to a discrete distribution. ${ }^{23}$ In this model, the charge dynamics can be described by a functional dependence of the form

$$
I(t)=I_{\mathrm{eq}}\left(1+C \exp \left\{\frac{-4 \pi a^{3} n}{3}[\ln (\nu t)]^{3}\right\}\right)
$$

Here, $n$ is the density of trapping defects, and $\nu$ and $a$ are parameters linked to the $\mathrm{NV}^{-}$center, defined as the tunneling frequency and the attenuation length of the wavefunction, respectively. Using this expression for the fits, we find $a=0.1$ $\mathrm{nm}, \nu=0.8 \pm 0.1 \mathrm{MHz}$, and $n=2-4 \mathrm{ppm}$. The density $n$ shows a slight tendency to decrease after long laser pulses, possibly indicating that photoexcited electrons have filled some charge traps. Additional work will be necessary to understand the features of $n$ and some subtleties not captured by the 
present model, such as nonuniform values of $n, \nu$, and $a$ across a defective sample. However, for our data, we do not observe a substantial improvement of the fit using eq 2 compared with a stretched exponential function (eq 1). We are mostly interested in the initial out-of-equilibrium values of the curves compared to equilibrium. These values are set during the initialization phase by the laser pulse length and power and do not depend critically on the choice of the fitting function. In this respect, the values returned by the two fitting procedures are in good agreement.

The leakage of the $\mathrm{NV}^{0}$ FL component in the $\mathrm{NV}^{-}$spectral region can affect the ODMR contrast. ${ }^{67-69}$ For $\mathrm{NV}^{-}$centers, the contrast is defined as $C_{s}=\left(I_{\text {off }}-I_{\text {on }}\right) / I_{\text {off }}$, where $I_{\text {off }}$ and $I_{\text {on }}$ are the collected FL intensities with microwaves off- and onresonance, respectively. If the intensity contains an $\mathrm{NV}^{0}$-related component $I_{0}$, then the contrast $C_{\mathrm{s}}$ must be scaled by a factor $I_{\text {off }} /\left(I_{\text {off }}+I_{0}\right)$. In our case, the intensity $I_{\text {off }}\left(I_{0}\right)$ is the fraction $f^{-}$ $\left(f^{0}\right)$ of the total $\mathrm{NV}^{-}\left(\mathrm{NV}^{0}\right)$ intensity filtered by the $>750 \mathrm{~nm}$ longpass and normalized by photon-counter efficiency. It is possible to relate these intensities to the charge-state ratio $R$ by parameters pertaining to $\mathrm{NV}^{-}\left(\mathrm{NV}^{0}\right)$ centers, such as the absorption cross sections $\sigma^{-}\left(\sigma^{0}\right)$, and the excited-state lifetime $\tau^{-}\left(\tau^{0}\right)$. The ODMR contrast for the NV ensemble is

$$
C_{\text {ens }}=C_{\mathrm{s}} \frac{R}{R+F}
$$

where $F=\frac{f^{0}}{f^{-}} \frac{\sigma^{0}}{\sigma^{-}} \frac{\tau^{-}}{\tau^{0}}$ is a numerical factor that depends on detection conditions, such as the choice of filters and detectors, and structural properties of NV centers. Conversely, $R$ can vary for different samples. For shallow NVs, where the charge state is dominated by neutral centers, $R$ is small and the ensemble contrast is substantially reduced. On the contrary, for samples that mostly contain $\mathrm{NV}^{-}$centers (as seen usually for bulk NV centers), $R$ is much larger than $F$ and the correction is minimal. For our setup, we estimate $f^{0} / f^{-} \approx 0.42$ from the shape of fluorescence (see Section S1). Using $\sigma^{-}=3.1 \times 10^{-17} \mathrm{~cm}^{2}, 70$ $\sigma^{0}=1.8 \times 10^{-17} \mathrm{~cm}^{2},{ }^{71} \tau^{-}=12 \mathrm{~ns}$, and $\tau^{0}=21 \mathrm{~ns},{ }^{72}$ we find $F$ $\approx 0.14$. Also, the charge state ratio varies dramatically from 0.15 at low laser power to 1.13 at high power (Figure $5 b, c$ ). With an indicative value of $C_{s}=10 \%$, we estimate ensemble contrasts of 5.2 and $8.9 \%$ at low and high laser powers, respectively, and a tendency to increase with $R$ and with laser power, following the $\mathrm{NV}^{0} \rightarrow \mathrm{NV}^{-}$photoconversion. In principle, $C_{s}$ can be recovered a posteriori from experimental values of the ODMR contrast, provided that the dependence of $R$ on the laser power is known. The proposed interpretation explains the trend shown in Figure $6 \mathrm{~b}$ from low to moderately high laser powers, although the contrast shows a saturation or even a slight reduction at the highest power levels (see, for instance, sample F2). It also explains the striking difference between the flat and the nanostructured samples: in the latter, $\mathrm{NV}^{0} \mathrm{~s}$ are by far the dominating species even at high laser power and the extracted contrast is always poorer than in the flat samples. Concerning the saturation of the contrast at high powers, we note that an optimal contrast results from combined laser and microwave spin initialization. If the laser power is too high, the microwave field cannot compete in inverting the population levels in the ground state, and some degree of laser-induced repolarization is expected. ${ }^{49}$ Despite setting the power of the microwave to a maximum available value (output of $8 \mathrm{dBm}$ amplified by $\approx 35 \mathrm{~dB}$ ), the laser repolarization effect may prevail, resulting in the saturation and slight reduction shown in Figure $6 \mathrm{~b}$.

The resulting increase in ODMR contrast improves sensitivity $\eta$ (expressed in $T / \sqrt{\mathrm{Hz}}$ ) and the minimum magnetic field $\delta B$ detectable at time $\Delta t$, defined as $\delta B=\eta / \sqrt{\Delta t}$. In continuous-wave ODMR protocols, sensitivity is given by the relation ${ }^{73}$

$$
\eta=\frac{4}{3 \sqrt{3}} \frac{h}{g \mu_{\mathrm{B}}} \frac{\Gamma}{C_{\mathrm{ens}} \sqrt{r}}
$$

where the numerical factor in front is related to the Lorentzian function used to fit the resonance, $h$ is the Planck constant, $\mu_{\mathrm{B}}$ is the Bohr magneton, $g \approx 2, \Gamma$ is the full width at halfmaximum of the Lorentzian, and $r$ is the photodetection rate. The contrast $C_{\text {ens }}$ in eq 4 is the ODMR contrast of the NV ensemble given by eq 3 . At low laser power $(50 \mathrm{~mW})$, we find experimentally $r=0.73 \times 10^{6} \mathrm{~s}^{-1}$, while at high power $(1500$ $\mathrm{mW}$ ), we find $r=17.8 \times 10^{6} \mathrm{~s}^{-1}$ (values normalized by limited photon counter efficiency of the detector). Taking values of $C_{\text {ens }}$ and $R$ as before, and a representative $\Gamma=10 \mathrm{MHz}$, we find $6.2 \mu \mathrm{T} / \sqrt{\mathrm{Hz}}$ and $730 \mathrm{nT} / \sqrt{\mathrm{Hz}}$ at low and high powers, respectively. Sensitivity might be further improved with the use of pulsed-wave ODMR ${ }^{73}$ and extended to the detection of AC magnetic fields. ${ }^{74,75}$ Therefore, magnetometry applications benefit from the use of strong laser power by an increase in the photon detection rate and improved contrast. We note that the correction factor of eq 3 does not exclusively determine the ODMR contrast but plays a role in other detection schemes, including pulsed sequences as those in Figure 3a,c.

${ }^{13} \mathrm{C}$ nuclear hyperpolarization is another technique that could benefit from an increased fraction of $\mathrm{NV}^{-} \mathrm{s}$. This technique is based on the transfer of spin polarization from highly polarized $\mathrm{NV}^{-}$to first-neighbor ${ }^{13} \mathrm{C}$ nuclei via spin-spin interaction at specific values of the applied magnetic field. ${ }^{76}$ Polarization can then diffuse through the diamond lattice to the bulk ${ }^{13} \mathrm{C}$ spin reservoir by nuclear spin-spin interactions. ${ }^{77,78}$ However, this process is much slower compared to the fast hyperpolarization of the first shell ${ }^{13} \mathrm{C}$ nuclei $(\approx 1 \mathrm{~ms})$ in the vicinity of $\mathrm{NV}^{-}$centers, and increasing the amount of $\mathrm{NV}^{-}$s via photoconversion can substantially improve the efficiency of the hyperpolarization process.

The effects reported here may be particularly important for hyperpolarization of nuclei outside the diamond surface. Indeed, it has been proposed that shallow $\mathrm{NV}^{-}$may provide a source of spin polarization of molecular moieties adsorbed at the diamond surface, ${ }^{16,17,79}$ thus complementing techniques like dynamic nuclear polarization or optical pumping spinexchange. Increasing their concentration in the vicinity, the diamond surface may enable hyperpolarization of nuclei outside the diamond lattice.

Realistically, laser power is constrained by the available equipment and by the nature of the sample. Some applications of sensing, ${ }^{80}$ widefield imaging, ${ }^{81}$ and hyperpolarization ${ }^{17}$ necessarily address a large number of NVs over broad twodimensional (2D) or three-dimensional (3D) regions at the expense of a tighter focusing (so, with lower laser intensity). Nevertheless, our results indicate that charge-state photoconversion must be taken into account for shallow NV centers and point to the maximization of laser power for improved $\mathrm{NV}^{-}$concentration and lifetime whenever possible.

In conclusion, we studied the effects of laser power on charge-state photoconversion in ensembles of shallow $\mathrm{NV}$ 
centers in flat and nanostructured diamonds. Surface effects favor the neutral charge state $\left(\mathrm{NV}^{0}\right)$ over the negative state $\left(\mathrm{NV}^{-}\right)$. This problem is exacerbated in the nanostructured samples. We show that high-power laser irradiation promotes $\mathrm{NV}^{0}$ to $\mathrm{NV}^{-}$photoconversion, thus significantly increasing the relative abundance of $\mathrm{NV}^{-} \mathrm{s}$ in flat and in a nanostructured diamond. Interestingly, we find that the excess $\mathrm{NV}^{-}$population is stable over timescales of tens or hundreds of milliseconds. This may provide an important advantage for magnetometry and polarization-transfer applications, where large ensembles of shallow $\mathrm{NV}^{-}$are needed.

\section{ASSOCIATED CONTENT}

\section{(s) Supporting Information}

The Supporting Information is available free of charge at https://pubs.acs.org/doi/10.1021/acsami.1c09825.

Charge state fluorescence ratio and filtering; effect of the readout pulse; excited state saturation; and calculation of the charged state ratio (PDF)

\section{AUTHOR INFORMATION}

\section{Corresponding Author}

Federico Gorrini - Istituto Italiano di Tecnologia, Center for Sustainable Future Technologies, 10144 Torino, Italy; Molecular Biology Center, University of Torino, 10126 Torino, Italy; 10 orcid.org/0000-0002-6420-4104; Email: federico.gorrini@iit.it

\section{Authors}

Carla Dorigoni - Istituto Italiano di Tecnologia, Center for Neuroscience and Cognitive System, 38068 Rovereto (Tn), Italy

Domingo Olivares-Postigo - Molecular Biology Center and Department of Molecular Biotechnology and Health Sciences, University of Torino, 10126 Torino, Italy; Istituto Italiano di Tecnologia, Center for Neuroscience and Cognitive System, 38068 Rovereto ( $\mathrm{Tn})$, Italy

Rakshyakar Giri - Istituto Italiano di Tecnologia, Center for Neuroscience and Cognitive System, 38068 Rovereto (Tn), Italy

Pietro Aprà - Department of Physics and "NIS Interdepartmental Centre", University of Torino, 10125 Torino, Italy; National Institute of Nuclear Physics, Torino 10125, Italy

Federico Picollo - Department of Physics and "NIS Interdepartmental Centre", University of Torino, 10125 Torino, Italy; National Institute of Nuclear Physics, Torino 10125, Italy; (1) orcid.org/0000-0003-3179-271X

Angelo Bifone - Istituto Italiano di Tecnologia, Center for Sustainable Future Technologies, 10144 Torino, Italy; Molecular Biology Center and Department of Molecular Biotechnology and Health Sciences, University of Torino, 10126 Torino, Italy

Complete contact information is available at: https://pubs.acs.org/10.1021/acsami.1c09825

\section{Notes}

The authors declare no competing financial interest.

\section{ACKNOWLEDGMENTS}

The authors thank P. Olivero for useful suggestions on sample treatments from the Department of Physics, University of
Torino, Italy; L. Basso for fluorescence spectroscopy with the micro-Raman setup from the Department of Physics, Università di Trento, Italy; and Simone Lauciello and Alice Scarpellini of Electron Microscopy Facility of Istituto Italiano di Tecnologia in Genova for SEM characterization. This project has received funding from the European Union's Horizon 2020 research and innovation programme under grant agreement No 858149 (AlternativesToGd) and from the Marie Skłodowska-Curie program, Grant Agreement No. 766402 (ZULF).

\section{REFERENCES}

(1) Kucsko, G.; Maurer, P. C.; Yao, N. Y.; Kubo, M.; Noh, H. J.; Lo, P. K.; Park, H.; Lukin, M. D. Nanometre-Scale Thermometry in a Living Cell. Nature 2013, 500, 54-58.

(2) Singam, S. K. R.; Nesladek, M.; Goovaerts, E. Nitrogen-Vacancy Nanodiamond Based Local Thermometry Using Frequency-Jump Modulation. Nanotechnology 2020, 31, No. 105501.

(3) Davis, H. C.; Ramesh, P.; Bhatnagar, A.; Lee-Gosselin, A.; Barry, J. F.; Glenn, D. R.; Walsworth, R. L.; Shapiro, M. G. Mapping the Microscale Origins of Magnetic Resonance Image Contrast with Subcellular Diamond Magnetometry. Nat. Commun. 2018, 9, No. 131.

(4) Schloss, J. M.; Barry, J. F.; Turner, M. J.; Walsworth, R. L. Simultaneous Broadband Vector Magnetometry Using Solid-State Spins. Phys. Rev. Appl. 2018, 10, No. 034044.

(5) Iwasaki, T.; Naruki, W.; Tahara, K.; Makino, T.; Kato, H.; Ogura, M.; Takeuchi, D.; Yamasaki, S.; Hatano, M. Direct Nanoscale Sensing of the Internal Electric Field in Operating Semiconductor Devices Using Single Electron Spins. ACS Nano 2017, 11, 12381245.

(6) Forneris, J.; Ditalia Tchernij, S.; Traina, P.; Moreva, E.; Skukan, N.; Jakšić, M.; Grilj, V.; Bosia, F.; Enrico, E.; Amato, G.; Degiovanni, I. P.; Naydenov, B.; Jelezko, F.; Genovese, M.; Olivero, P. Mapping the Local Spatial Charge in Defective Diamond by Means of NV Sensors - A "Self-Diagnostic" Concept. Phys. Rev. Appl. 2018, 10, No. 014024.

(7) Tetienne, J. P.; Lombard, A.; Simpson, D. A.; Ritchie, C.; Lu, J.; Mulvaney, P.; Hollenberg, L. C. L. Scanning Nanospin Ensemble Microscope for Nanoscale Magnetic and Thermal Imaging. Nano Lett. 2016, 16, 326-333.

(8) Gorrini, F.; Giri, R.; Avalos, C. E.; Tambalo, S.; Mannucci, S.; Basso, L.; Bazzanella, N.; Dorigoni, C.; Cazzanelli, M.; Marzola, P.; Miotello, A.; Bifone, A. Fast and Sensitive Detection of Paramagnetic Species Using Coupled Charge and Spin Dynamics in Strongly Fluorescent Nanodiamonds. ACS Appl. Mater. Interfaces 2019, 11, 24412-24422.

(9) Wang, P.; Chen, S.; Guo, M.; Peng, S.; Wang, M.; Chen, M.; Ma, W.; Zhang, R.; Su, J.; Rong, X.; Shi, F.; Xu, T.; Du, J. Nanoscale Magnetic Imaging of Ferritins in a Single Cell. Sci. Adv. 2019, 5, No. eaau8038.

(10) Radu, V.; Price, J. C.; Levett, S. J.; Narayanasamy, K. K.; Bateman-Price, T. D.; Wilson, P. B.; Mather, M. L. Dynamic Quantum Sensing of Paramagnetic Species Using Nitrogen-Vacancy Centers in Diamond. ACS Sens. 2019, 5, 703-710.

(11) Balasubramanian, G.; Lazariev, A.; Arumugam, S. R.; Duan, D. wen. Nitrogen-Vacancy Color Center in Diamond-Emerging Nanoscale Applications in Bioimaging and Biosensing. Curr. Opin. Chem. Biol. 2014, 20, 69-77.

(12) Hsiao, W. W. W.; Hui, Y. Y.; Tsai, P. C.; Chang, H. C. Fluorescent Nanodiamond: A Versatile Tool for Long-Term Cell Tracking, Super-Resolution Imaging, and Nanoscale Temperature Sensing. Acc. Chem. Res. 2016, 49, 400-407.

(13) Boretti, A.; Rosa, L.; Blackledge, J.; Castelletto, S. NitrogenVacancy Centers in Diamond for Nanoscale Magnetic Resonance Imaging Applications. Beilstein J. Nanotechnol. 2019, 10, 2128-2151.

(14) King, J. P.; Jeong, K.; Vassiliou, C. C.; Shin, C. S.; Page, R. H.; Avalos, C. E.; Wang, H. J.; Pines, A. Room-Temperature in Situ 
Nuclear Spin Hyperpolarization from Optically Pumped Nitrogen Vacancy Centres in Diamond. Nat. Commun. 2015, 6, No. 8965.

(15) Alvarez, G. A.; Bretschneider, C. O.; Fischer, R.; London, P.; Kanda, H.; Onoda, S.; Isoya, J.; Gershoni, D.; Frydman, L. Local and Bulk 13 C Hyperpolarization in Nitrogen-Vacancy-Centred Diamonds at Variable Fields and Orientations. Nat. Commun. 2015, 6, No. 8456.

(16) Ajoy, A.; Liu, K.; Nazaryan, R.; Lv, X.; Zangara, P. R.; Safvati, B.; Wang, G.; Arnold, D.; Li, G.; Lin, A.; Raghavan, P.; Druga, E.; Dhomkar, S.; Pagliero, D.; Reimer, J. A.; Suter, D.; Pines, A.; Meriles, C. A. Orientation-Independent Room Temperature Optical 13C Hyperpolarization in Powdered Diamond. Sci. Adv. 2018, 4, No. eaar5492.

(17) Healey, A. J.; Hall, L. T.; White, G. A. L.; Teraji, T.; Sani, M.A.; Separovic, F.; Tetienne, J.-P.; Hollenberg, L. C. L. Polarization Transfer to External Nuclear Spins Using Ensembles of NitrogenVacancy Centers. Phys. Rev. Appl. 2021, 15, No. 054052.

(18) Neu, E.; Appel, P.; Ganzhorn, M.; Miguel-Sánchez, J.; Lesik, M.; Mille, V.; Jacques, V.; Tallaire, A.; Achard, J.; Maletinsky, P. Photonic Nano-Structures on (111)-Oriented Diamond. Appl. Phys. Lett. 2014, 104, No. 153108.

(19) Alghannam, F.; Hemmer, P. Engineering of Shallow Layers of Nitrogen Vacancy Colour Centres in Diamond Using Plasma Immersion Ion Implantation. Sci. Rep. 2019, 9, No. 5870.

(20) Andrich, P.; Li, J.; Liu, X.; Heremans, F. J.; Nealey, P. F.; Awschalom, D. D. Microscale Resolution Thermal Mapping Using a Flexible Platform of Patterned Quantum Sensors. Nano Lett. 2018, 18, 4684-4690.

(21) Aslam, N.; Waldherr, G.; Neumann, P.; Jelezko, F.; Wrachtrup, J. Photo-Induced Ionization Dynamics of the Nitrogen Vacancy Defect in Diamond Investigated by Single-Shot Charge State Detection. New J. Phys. 2013, 15, No. 013064.

(22) Chen, X. D.; Zou, C. L.; Sun, F. W.; Guo, G. C. Optical Manipulation of the Charge State of Nitrogen-Vacancy Center in Diamond. Appl. Phys. Lett. 2013, 103, No. 013112.

(23) Dhomkar, S.; Jayakumar, H.; Zangara, P. R.; Meriles, C. A. Charge Dynamics in Near-Surface, Variable-Density Ensembles of Nitrogen-Vacancy Centers in Diamond. Nano Lett. 2018, 18, 40464052.

(24) Giri, R.; Gorrini, F.; Dorigoni, C.; Avalos, C. E.; Cazzanelli, M.; Tambalo, S.; Bifone, A. Coupled Charge and Spin Dynamics in HighDensity Ensembles of Nitrogen-Vacancy Centers in Diamond. Phys. Rev. B 2018, 98, No. 045401.

(25) Bluvstein, D.; Zhang, Z.; Jayich, A. C. B. Identifying and Mitigating Charge Instabilities in Shallow Diamond Nitrogen-Vacancy Centers. Phys. Rev. Lett. 2019, 122, No. 076101.

(26) Cui, S.; Hu, E. L. Increased Negatively Charged NitrogenVacancy Centers in Fluorinated Diamond. Appl. Phys. Lett. 2013, 103, No. 051603 .

(27) Murai, T.; Makino, T.; Kato, H.; Shimizu, M.; Murooka, T.; Herbschleb, E. D.; Doi, Y.; Morishita, H.; Fujiwara, M.; Hatano, M.; Yamasaki, S.; Mizuochi, N. Engineering of Fermi Level by Nin Diamond Junction for Control of Charge States of NV Centers. Appl. Phys. Lett. 2018, 112, No. 111903.

(28) Forneris, J.; Ditalia Tchernij, S.; Tengattini, A.; Enrico, E.; Grilj, V.; Skukan, N.; Amato, G.; Boarino, L.; Jakšíc, M.; Olivero, P. Electrical Control of Deep NV Centers in Diamond by Means of SubSuperficial Graphitic Micro-Electrodes. Carbon 2017, 113, 76-86.

(29) Trusheim, M. E.; Li, L.; Laraoui, A.; Chen, E. H.; Bakhru, H.; Schröder, T.; Gaathon, O.; Meriles, C. A.; Englund, D. Scalable Fabrication of High Purity Diamond Nanocrystals with Long-SpinCoherence Nitrogen Vacancy Centers. Nano Lett. 2014, 14, 32-36.

(30) Naydenov, B.; Reinhard, F.; Lämmle, A.; Richter, V.; Kalish, R.; D'Haenens-Johansson, U. F. S.; Newton, M.; Jelezko, F.; Wrachtrup, J. Increasing the Coherence Time of Single Electron Spins in Diamond by High Temperature Annealing. Appl. Phys. Lett. 2010, 97, No. 242511.
(31) Toyli, D. M.; Weis, C. D.; Fuchs, G. D.; Schenkel, T.; Awschalom, D. D. Chip-Scale Nanofabrication of Single Spins and Spin Arrays in Diamond. Nano Lett. 2010, 10, 3168-3172.

(32) Spinicelli, P.; Dréau, A.; Rondin, L.; Silva, F.; Achard, J.; Xavier, S.; Bansropun, S.; Debuisschert, T.; Pezzagna, S.; Meijer, J.; Jacques, V.; Roch, J. F. Engineered Arrays of Nitrogen-Vacancy Color Centers in Diamond Based on Implantation of $\mathrm{Cn}$ ? Molecules through Nanoapertures. New J. Phys. 2011, 13, No. 025014.

(33) Ziegler, J. F.; Ziegler, M. D.; Biersack, J. P. The Stopping and Range of Ions in Matter. Nucl. Instrum. Methods Phys. Res., Sect. B 2010, 268, 1818-1823.

(34) Antonov, D.; Häuermann, T.; Aird, A.; Roth, J.; Trebin, H. R.; Müller, C.; McGuinness, L.; Jelezko, F.; Yamamoto, T.; Isoya, J.; Pezzagna, S.; Meijer, J.; Wrachtrup, J. Statistical Investigations on Nitrogen-Vacancy Center Creation. Appl. Phys. Lett. 2014, 104, No. 012105.

(35) Pezzagna, S.; Naydenov, B.; Jelezko, F.; Wrachtrup, J.; Meijer, Jjnj. Creation Efficiency of Nitrogen-Vacancy Centres in Diamond. New J. Phys. 2010, 12, No. 065017.

(36) Giri, R.; Dorigoni, C.; Tambalo, S.; Gorrini, F.; Bifone, A. Selective Measurement of Charge Dynamics in an Ensemble of Nitrogen-Vacancy Centers in Nano- and Bulk Diamonds. Phys. Rev. B 2019, 99, No. 155426.

(37) Doherty, M. W.; Manson, N. B.; Delaney, P.; Jelezko, F.; Wrachtrup, J.; Hollenberg, L. C. L. The Nitrogen-Vacancy Colour Centre in Diamond. Phys. Rep. 2013, 528, 1-45.

(38) Bourgeois, E.; Gulka, M.; Nesladek, M. Photoelectric Detection and Quantum Readout of Nitrogen-Vacancy Center Spin States in Diamond. Adv. Opt. Mater. 2020, 8, No. 1902132.

(39) Manson, N. B.; Hedges, M.; Barson, M. S. J.; Ahlefeldt, R.; Doherty, M. W.; Abe, H.; Ohshima, T.; Sellars, M. J. NV- - N+ Pair Centre in 1b Diamond. New J. Phys. 2018, 20, No. 113037.

(40) Alsid, S. T.; Barry, J. F.; Pham, L. M.; Schloss, J. M.; O’Keeffe, M. F.; Cappellaro, P.; Braje, D. A. Photoluminescence Decomposition Analysis: A Technique to Characterize N-V Creation in Diamond. Phys. Rev. Appl. 2019, 12, No. 044003.

(41) Tetienne, J. P.; Rondin, L.; Spinicelli, P.; Chipaux, M.; Debuisschert, T.; Roch, J. F.; Jacques, V. Magnetic-Field-Dependent Photodynamics of Single NV Defects in Diamond: An Application to Qualitative All-Optical Magnetic Imaging. New J. Phys. 2012, 14, No. 103033.

(42) Capelli, M.; Reineck, P.; Lau, D. W. M.; Orth, A.; Jeske, J.; Doherty, M. W.; Ohshima, T.; Greentree, A. D.; Gibson, B. C. Magnetic Field-Induced Enhancement of the Nitrogen-Vacancy Fluorescence Quantum Yield. Nanoscale 2017, 9, 9299-9304.

(43) Doherty, M. W.; Dolde, F.; Fedder, H.; Jelezko, F.; Wrachtrup, J.; Manson, N. B.; Hollenberg, L. C. L. Theory of the Ground-State Spin of the NV - Center in Diamond. Phys. Rev. B 2012, 85, No. 205203.

(44) Manson, N. B.; Harrison, J. P. Photo-Ionization of the Nitrogen-Vacancy Center in Diamond. Diam. Relat. Mater. 2005, 14, $1705-1710$.

(45) Acosta, V. M.; Bauch, E.; Ledbetter, M. P.; Santori, C.; Fu, K.M.; Barclay, P. E.; Beausoleil, R. G.; Linget, H.; Roch, J. F.; Treussart, F.; et al. Diamonds with a High Density of Nitrogen-Vacancy Centers for Magnetometry Applications. Phys. Rev. B 2009, 80, No. 115202.

(46) Kurtsiefer, C.; Mayer, S.; Zarda, P.; Weinfurter, H. Stable SolidState Source of Single Photons. Phys. Rev. Lett. 2000, 85, No. 290.

(47) Manson, N. B.; Harrison, J. P.; Sellars, M. J. Nitrogen-Vacancy Center in Diamond: Model of the Electronic Structure and Associated Dynamics. Phys. Rev. B 2006, 74, No. 104303.

(48) Plakhotnik, T.; Gruber, D. Luminescence of Nitrogen-Vacancy Centers in Nanodiamonds at Temperatures between 300 and $700 \mathrm{~K}$ : Perspectives on Nanothermometry. Phys. Chem. Chem. Phys. 2010, 12, 9751-9756.

(49) Jensen, K.; Acosta, V. M.; Jarmola, A.; Budker, D. Light Narrowing of Magnetic Resonances in Ensembles of NitrogenVacancy Centers in Diamond. Phys. Rev. B 2013, 87, No. 014115. 
(50) Santori, C.; Barclay, P. E.; Fu, K.-M. C.; Beausoleil, R. G. Vertical Distribution of Nitrogen-Vacancy Centers in Diamond Formed by Ion Implantation and Annealing. Phys. Rev. B 2009, 79, No. 125313.

(51) Hauf, M. V.; Grotz, B.; Naydenov, B.; Dankerl, M.; Pezzagna, S.; Meijer, J.; Jelezko, F.; Wrachtrup, J.; Stutzmann, M.; Reinhard, F.; Garrido, J. A. Chemical Control of the Charge State of NitrogenVacancy Centers in Diamond. Phys. Rev. B 2011, 83, No. 081304.

(52) Newell, A. N.; Dowdell, D. A.; Santamore, D. H. Surface Effects on Nitrogen Vacancy Centers Neutralization in Diamond. J. Appl. Phys. 2016, 120, No. 185104.

(53) Kaviani, M.; Deák, P.; Aradi, B.; Frauenheim, T.; Chou, J. P.; Gali, A. Proper Surface Termination for Luminescent Near-Surface NV Centers in Diamond. Nano Lett. 2014, 14, 4772-4777.

(54) Meirzada, I.; Hovav, Y.; Wolf, S. A.; Bar-Gill, N. Negative Charge Enhancement of Near-Surface Nitrogen Vacancy Centers by Multicolor Excitation. Phys. Rev. B 2018, 98, No. 245411.

(55) Gaebel, T.; Domhan, M.; Wittmann, C.; Popa, I.; Jelezko, F.; Rabeau, J.; Greentree, A.; Prawer, S.; Trajkov, E.; Hemmer, P. R.; Wrachtrup, J. Photochromism in Single Nitrogen-Vacancy Defect in Diamond. Appl. Phys. B 2006, 82, 243-246.

(56) Iakoubovskii, K.; Adriaenssens, G. J. Optical Transitions at the Substitutional Nitrogen Centre in Diamond. J. Phys.: Condens. Matter 2000, 12, L77.

(57) Jayakumar, H.; Henshaw, J.; Dhomkar, S.; Pagliero, D.; Laraoui, A.; Manson, N. B.; Albu, R.; Doherty, M. W.; Meriles, C. A. Optical Patterning of Trapped Charge in Nitrogen-Doped Diamond. Nat. Commun. 2016, 7, No. 12660.

(58) Fu, K.-M.; Santori, C.; Barclay, P. E.; Beausoleil, R. G. Conversion of Neutral Nitrogen-Vacancy Centers to Negatively Charged Nitrogen-Vacancy Centers through Selective Oxidation. Appl. Phys. Lett. 2010, 96, No. 121907.

(59) McCloskey, D. J.; Dontschuk, N.; Broadway, D. A.; Nadarajah, A.; Stacey, A.; Tetienne, J.-P.; Hollenberg, L. C. L.; Prawer, S.; Simpson, D. A. Enhanced Widefield Quantum Sensing with NitrogenVacancy Ensembles Using Diamond Nanopillar Arrays. ACS Appl. Mater. Interfaces 2020, 12, 13421-13427.

(60) Tallaire, A.; Mayer, L.; Brinza, O.; Pinault-Thaury, M.-A.; Debuisschert, T.; Achard, J. Highly Photostable NV Centre Ensembles in CVD Diamond Produced by Using N2O as the Doping Gas. Appl. Phys. Lett. 2017, 111, No. 143101.

(61) Meijer, J.; Burchard, B.; Domhan, M.; Wittmann, C.; Gaebel, T.; Popa, I.; Jelezko, F.; Wrachtrup, J. Generation of Single Color Centers by Focused Nitrogen Implantation. Appl. Phys. Lett. 2005, 87, No. 261909.

(62) Waldermann, F. C.; Olivero, P.; Nunn, J.; Surmacz, K.; Wang, Z. Y.; Jaksch, D.; Taylor, R. A.; Walmsley, I. A.; Draganski, M.; Reichart, P.; et al. Creating Diamond Color Centers for Quantum Optical Applications. Diam. Relat. Mater. 2007, 16, 1887-1895.

(63) Deák, P.; Aradi, B.; Kaviani, M.; Frauenheim, T.; Gali, A. Formation of NV Centers in Diamond: A Theoretical Study Based on Calculated Transitions and Migration of Nitrogen and Vacancy Related Defects. Phys. Rev. B 2014, 89, No. 075203.

(64) Choi, J.; Choi, S.; Kucsko, G.; Maurer, P. C.; Shields, B. J.; Sumiya, H.; Onoda, S.; Isoya, J.; Demler, E.; Jelezko, F.; Yao, N. Y.; Lukin, M. D. Depolarization Dynamics in a Strongly Interacting SolidState Spin Ensemble. Phys. Rev. Lett. 2017, 118, No. 093601.

(65) Miller, J. R. Scavenging Kinetics of Electrons Produced by Irradiation of Organic Glasses; Experimental Evidence for Long Range Tunneling. J. Chem. Phys. 1972, 56, 5173-5183.

(66) Tachiya, M.; Mozumder, A. Decay of Trapped Electrons by Tunnelling to Scavenger Molecules in Low-Temperature Glasses. Chem. Phys. Lett. 1974, 28, 87-89.

(67) Aude Craik, D. P. L.; Kehayias, P.; Greenspon, A. S.; Zhang, X.; Turner, M. J.; Schloss, J. M.; Bauch, E.; Hart, C. A.; Hu, E. L.; Walsworth, R. L. A Microwave-Assisted Spectroscopy Technique for Determining Charge State in Nitrogen-Vacancy Ensembles in Diamond. Phys. Rev. Appl. 2020, 14, No. 014009.
(68) Yuan, Z.; Fitzpatrick, M.; Rodgers, L. V. H.; Sangtawesin, S.; Srinivasan, S.; de Leon, N. P. Charge State Dynamics and Optically Detected Electron Spin Resonance Contrast of Shallow NitrogenVacancy Centers in Diamond. Phys. Rev. Res. 2020, 2, No. 033263.

(69) Hopper, D. A.; Lauigan, J. D.; Huang, T. Y.; Bassett, L. C. RealTime Charge Initialization of Diamond Nitrogen-Vacancy Centers for Enhanced Spin Readout. Phys. Rev. Appl. 2020, 13, No. 024016.

(70) Wee, T. L.; Tzeng, Y. K.; Han, C. C.; Chang, H. C.; Fann, W.; Hsu, J. H.; Chen, K. M.; Yu, E. C. Two-Photon Excited Fluorescence of Nitrogen-Vacancy Centers in Proton-Irradiated Type Ib Diamond. J. Phys. Chem. A 2007, 111, 9379-9386.

(71) Fraczek, E.; Savitski, V. G.; Dale, M.; Breeze, B. G.; Diggle, P.; Markham, M.; Bennett, A.; Dhillon, H.; Newton, M. E.; Kemp, A. J. Laser Spectroscopy of NV- and NV0 Colour Centres in Synthetic Diamond. Opt. Mater. Express 2017, 7, No. 02571.

(72) Storteboom, J.; Dolan, P.; Castelletto, S.; Li, X.; Gu, M. Lifetime Investigation of Single Nitrogen Vacancy Centres in Nanodiamonds. Opt. Express 2015, 23, No. 011327.

(73) Dréau, A.; Lesik, M.; Rondin, L.; Spinicelli, P.; Arcizet, O.; Roch, J. F.; Jacques, V. Avoiding Power Broadening in Optically Detected Magnetic Resonance of Single NV Defects for Enhanced Dc Magnetic Field Sensitivity. Phys. Rev. B 2011, 84, No. 195204.

(74) Taylor, J. M.; Cappellaro, P.; Childress, L.; Jiang, L.; Budker, D.; Hemmer, P. R.; Yacoby, A.; Walsworth, R.; Lukin, M. D. HighSensitivity Diamond Magnetometer with Nanoscale Resolution. Nat. Phys. 2008, 4, 810-816.

(75) Barry, J. F.; Schloss, J. M.; Bauch, E.; Turner, M. J.; Hart, C. A.; Pham, L. M.; Walsworth, R. L. Sensitivity Optimization for NVDiamond Magnetometry. Rev. Mod. Phys. 2020, 92, No. 015004.

(76) Fischer, R.; Bretschneider, C. O.; London, P.; Budker, D.; Gershoni, D.; Frydman, L. Bulk Nuclear Polarization Enhanced at Room Temperature by Optical Pumping. Phys. Rev. Lett. 2013, 111, No. 057601.

(77) Ajoy, A.; Safvati, B.; Nazaryan, R.; Oon, J. T.; Han, B.; Raghavan, P.; Nirodi, R.; Aguilar, A.; Liu, K.; Cai, X.; Lv, X.; Druga, E.; Ramanathan, C.; Reimer, J. A.; Meriles, C. A.; Suter, D.; Pines, A. Hyperpolarized Relaxometry Based Nuclear T1 Noise Spectroscopy in Hybrid Diamond Quantum Registers. Nat. Commun. 2019, 10, No. 5160.

(78) Parker, A. J.; Jeong, K.; Avalos, C. E.; Hausmann, B. J. M.; Vassiliou, C. C.; Pines, A.; King, J. P. Optically Pumped Dynamic Nuclear Hyperpolarization in C 13 -Enriched Diamond. Phys. Rev. B 2019, 100, No. 041203.

(79) Abrams, D.; Trusheim, M. E.; Englund, D. R.; Shattuck, M. D.; Meriles, C. A. Dynamic Nuclear Spin Polarization of Liquids and Gases in Contact with Nanostructured Diamond. Nano Lett. 2014, 14, 2471-2478.

(80) Wolf, T.; Neumann, P.; Nakamura, K.; Sumiya, H.; Ohshima, T.; Isoya, J.; Wrachtrup, J. Subpicotesla Diamond Magnetometry. Phys. Rev. X 2015, 5, No. 041001.

(81) Tetienne, J.-P.; Dontschuk, N.; Broadway, D. A.; Stacey, A.; Simpson, D. A.; Hollenberg, L. C. L. Quantum Imaging of Current Flow in Graphene. Sci. Adv. 2017, 3, No. e1602429. 